\title{
Bearing only Target Tracking using Single and Multisensor: A Review
}

\author{
B. Sindhu ${ }^{1}$, J. Valarmathi ${ }^{1, *}$ and S. Christopher ${ }^{2}$ \\ ${ }^{I}$ School of Electronics Engineering, Vellore Institute of Technology, Vellore, Tamil Nadu, India. \\ ${ }^{2}$ DRDO, Delhi, India
}

Received 6 April 2018; Accepted 17 February 2019

\begin{abstract}
The brief review of methods used for estimating the target state in single and multi-sensor bearing only tracking (BOT) is presented in this paper. It deals with the target state estimation using bearing only measurements. BOT is difficult because of its poor observability in target state and nonlinearity in measurements. The complete survey is done on existing techniques, involved to overcome the difficulties caused by BOT. Here, the target tracking scenarios are divided into three different categories based on the nature of target motion and the number of target and sensors involved. The existing techniques involved are reviewed in detail. Finally the future trends for BOT are also discussed.
\end{abstract}

Keywords: Bearing only tracking, maneuvering target, nonlinear filters, multisensor-multitarget tracking.

\section{Introduction}

BOT has been an active research area for several years due to the challenges involved in it $[2,4]$. It is the widely used method in many applications like radar, sonar based navigation, underwater, space surveillance, ballistic trajectory estimation, submarine tracking using passive sonar, infrared (IR) sensors, wireless sensor networks and missile guidance $[3,50,65,15,29,41]$. BOT involves estimating the target state from noisy bearing measurements $[83,87,10,105]$.

The issue of poor observability caused due to BOT was reduced by taking an appropriate ownship maneuver and its dynamics should be higher degree than that of the target's dynamics to gain the observability of target state $[68,30,31,19]$. The necessary conditions for observability of target state were derived and discussed in $[21,51,85,68$, 30]. Using the bearing measurements there exist many techniques to estimate the target state $[45,53]$. If the measurements are linear, then basic Kalman filter is sufficient to estimate the target state $[1,23]$. If it is nonlinear, then special care has to be taken to linearize the nonlinear measurements. Thus for nonlinear measurements, different types of algorithms are available in the literature and are broadly classified into two types namely: batch processing and recursive Bayesian processing [28, 77]. In batch processing technique, set of measurements are considered for state estimation. On the other hand in recursive Bayesian method, each measurement is processed at a time recursively. Later one is suitable for most of real time systems due to its fast convergence [3, 45]. But choosing different algorithms not only depends on the target motion but also based on the scenarios. Thus in this paper we have considered three category to review the techniques. First two categories are based on the target motion (constant velocity

*E-mail address: jvalarmathi@vit.ac.in

ISSN: 1791-2377 @ 2019 Eastern Macedonia and Thrace Institute of Technology. All rights reserved. doi:10.25103/jestr.121.07 or constant acceleration) when there exist the single target and single ownship and third category is the extension of first two category when there exist multiple target and multiple ownship in the scenario.

\section{Category 1: Constant velocity target with maneuvering ownship}

In this category, both batch processing and recursive Bayesian approach can be used for the state estimation. Some of batch processing algorithms include maximum likelihood (ML) estimator, pseudo-linear (PL) estimator, batch-recursive estimator, batch maximum a posteriori (MAP) estimator [45, 37, 52]. Since recursive Bayesian approach is best suited for real time scenario, most of the time, this approach is preferred than batch processing. The most widely used recursive Bayesian filter for nonlinear measurements is an Extended Kalman filter formulated in Cartesian coordinates (EKF-Cart) [4,23]. The limitations in EKF-Cart leads to the formulation of modified spherical coordinates (EKF- MSC) [86] and log spherical coordinate (EKF- LSC) [2]. Other types of Kalman filters like Modified gain Extended Kalman filter (MGEKF) [6] and Unscented Kalman filter (UKF) can also be used for the nonlinear measurements. Other than this, some of the researchers recommended Particle filter (PF), Particle flow filter (PFF), pseudo linear Kalman filter (PLKF), and any of the nonlinear filters with multiple model approach (MM) [80,26]. The review of all these methods, their advantages and disadvantages are discussed briefly in section 2 .

Category 2: Constant acceleration target with maneuvering ownship

This category deals with the techniques involved in estimating the state of maneuvering target. Since batch processing cannot manage the target maneuvers, it is rarely recommended [7,45]. Hence, the most widely used technique is Interactive Multiple Model (IMM) along with nonlinear filters $[65,45]$. It works by switching between multiple dynamic models according to the target maneuver 
$[78,71]$. Depending upon the situation any one of the nonlinear filters mentioned in category 1 may be used along with IMM [78,65,71]. More detailed explanation of the techniques involved are reviewed briefly in section 3 .

Category 3: Multiple moving targets with multiple ownships (maneuvering/non-maneuvering target)

This category review the techniques involved in multiple moving targets with multiple sensors (ownship) irrespective of the target motion. Since multiple sensors are involved, observability is not a major issue [88, 99]. But tracking a particular target in a multiple moving target scenario makes the process complicated. Because, target detection by each sensor will be independent and the measurements are observed at random times, ambiguity may occur whether the observed data may originate from the target being tracked or from the clutter or any new target $[64,35]$. This will lead to the problem of measurement origin uncertainty [81]. To resolve these issues, batch processing algorithms like Maximum likelihood (ML) estimator and ML probabilistic data association (ML-PDA) are used. Since the batch processing techniques may not be applicable for scenarios involving three or more targets, the recursive algorithms are proposed that can integrate the received information from different sensors to identify the number of targets and its location in the surveillance region [88, 81]. The recursive algorithms include Multiframe assignment algorithm (MFA), Multi-target Multi-scan algorithm and Multiple hypotheses tracking (MHT) algorithm [81,64,32]. Each of these methods uses nonlinear filters mentioned in category 1 to estimate the target state. The more detailed explanations are given in section 4 .

In forthcoming sections this paper presents the complete survey on evolution of different algorithms applied to estimate the target state in BOT for the above mentioned three categories.

The outline of the paper is as follows. Section 2 describes the brief review of the techniques involved in category 1 . Section 3 concentrates the evolution of the techniques involved in state estimation for category 2 . Similarly, section 4 reviews the techniques involved in category 3 . Finally section 5 , gives the conclusion and future work.

\section{Review of techniques for category 1}

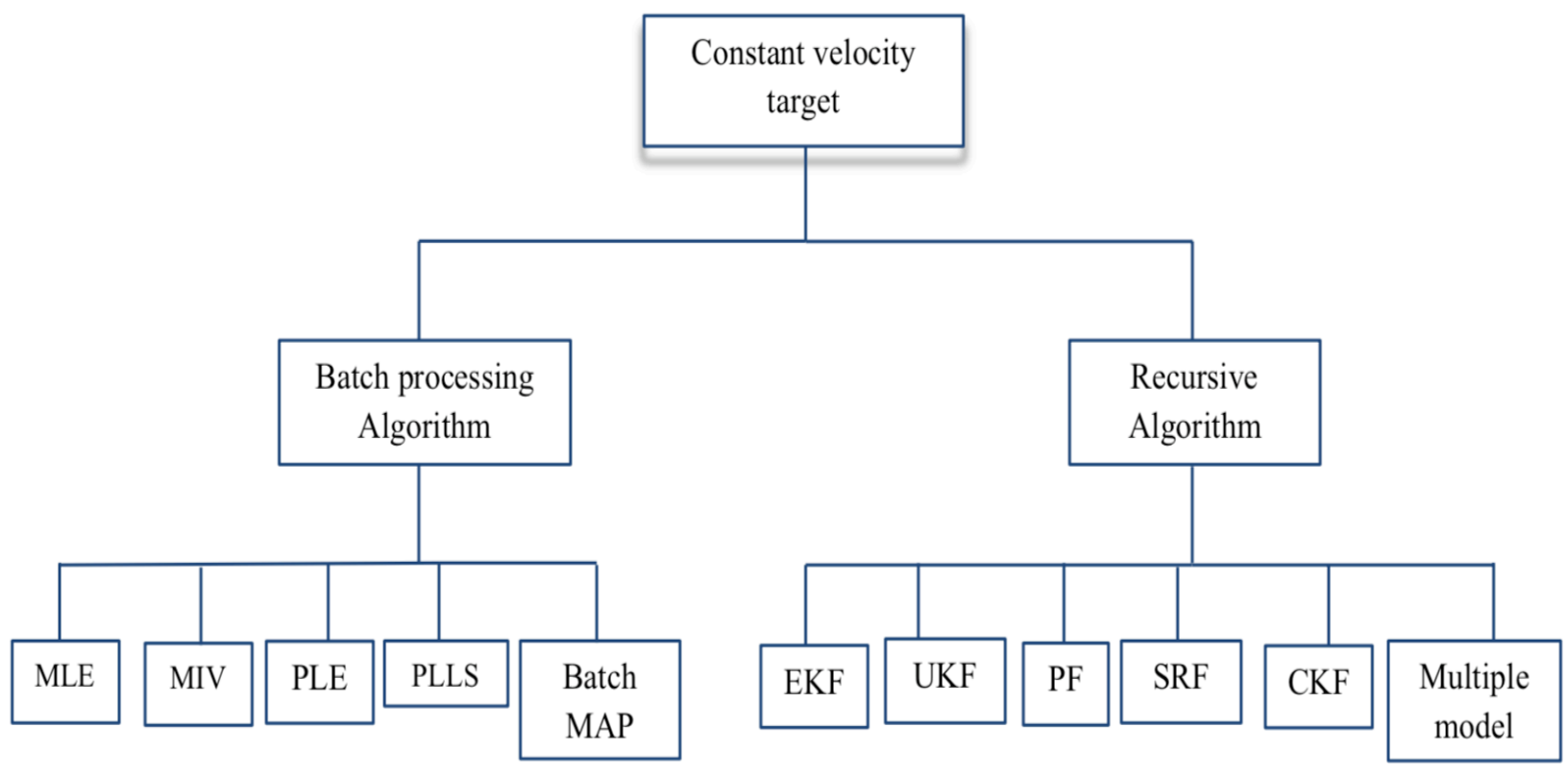

Fig.1. Techniques involved for constant velocity target.

$\begin{array}{lll}\text { - } & \text { MLE } & \text { - Maximum Likelihood estimate } \\ \text { - } & \text { MIV } & \text { - Modified-instrumental variable } \\ \text { - } & \text { PLE } & \text { - Pseudo-linear estimate } \\ \text { - } & \text { PLLS } & \text { - Pseudo linear least square } \\ \text { - } & \text { MAP } & \text { - Maximum a posteriori } \\ \text { - } & \text { EKF } & \text { - Extended Kalman filter } \\ \text { - } & \text { UKF } & \text { - Unscented Kalman filter } \\ \text { - } & \text { PF } & \text { - Particle filter } \\ \text { - } & \text { SRF } & \text { - Shifted Rayleigh filter } \\ \text { - } & \text { CKF } & \text { - Cubature Kalman filter }\end{array}$

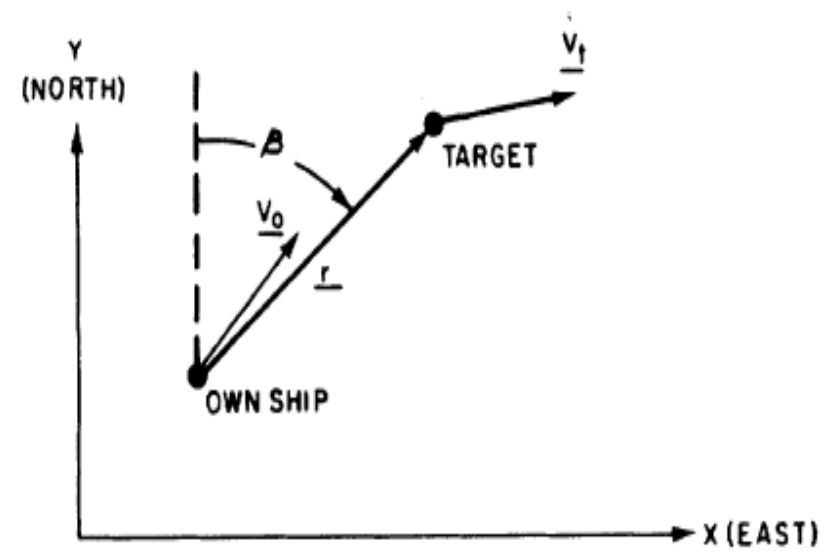

(a) 


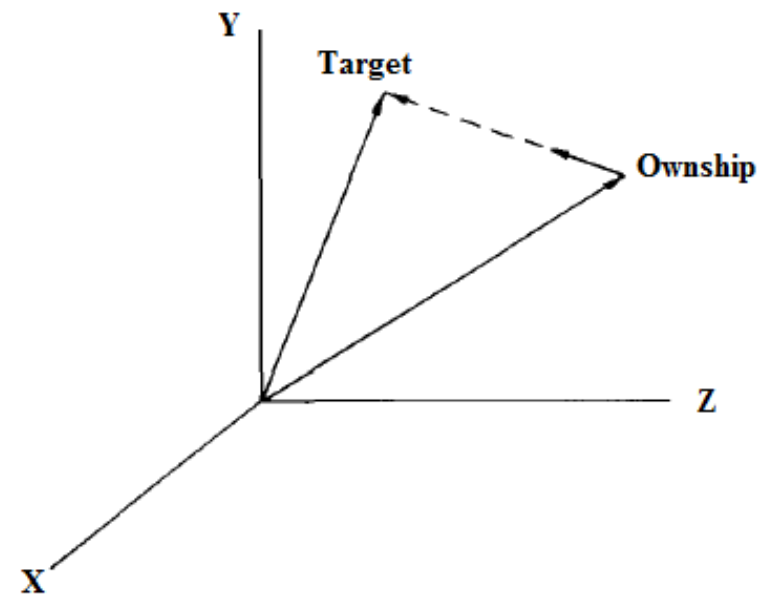

(b)

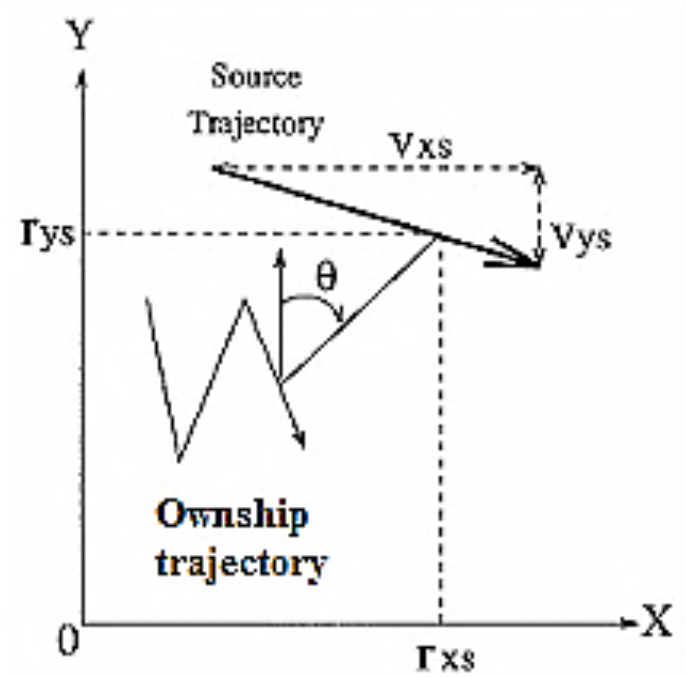

(c)

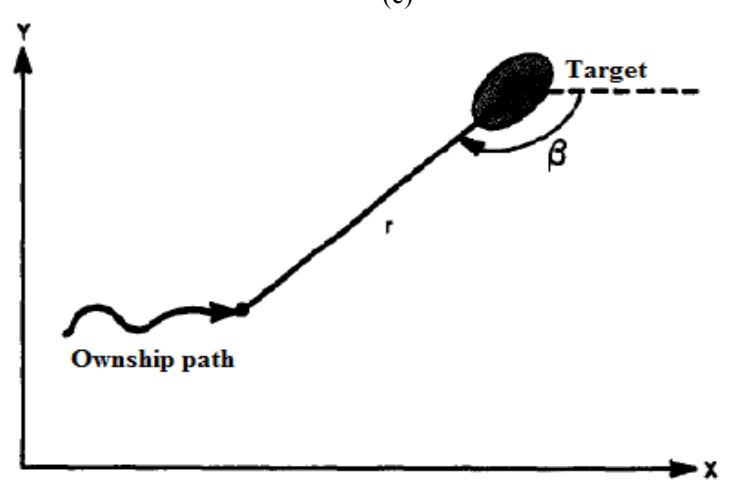

(d)

Fig.2. Maneuvering patterns of ownship to track single target when it is moving at constant velocity (Courtesy [2], [21], [56], [31]).

Fig. 1 shows the block diagram of various techniques used for category 1. Fig. 2 illustrates the different ownship maneuvering patterns to gain the observability of the target moving with constant velocity. For this category if the ownship does not move and change its velocity to take a maneuver, there will not be a relative velocity between the target and ownship. The relative motion between the target and ownship at each time will just be a linear trajectory with the fixed angle of arrival [106]. Hence the system will be unobservable, the relative position and velocity of target will not be determined. Thus the ownship takes different maneuver to gain observability in range and target state $[62,24,58]$ as shown in Fig.2. Following sections briefs the different techniques used for target state estimation in air space application and satellite application.

\subsection{Air space applications:}

BOT is widely used in air space applications for tracking aircrafts, ballistic trajectory tracking and satellite tracking. The techniques used in literature are explained briefly in the upcoming subsections.

\section{Target state estimation}

The observability requirements for BOT in air space are derived and explained briefly in [75, 30, 21, 85]. Same analysis for linear discrete time BOT is explained in [56]. It is a direct approach and uses simple linear algebraic formulation for observability analysis of a moving target. The possible ownship maneuvering patterns are shown in Fig. 2 and the necessary conditions for ownship maneuver was derived in $[30,56,68,85]$. Using this condition an optimal ownship maneuver is framed in [31,33] and they also proved the enhancement in system observability and accuracy through results [54]. Since, BOT is a nonlinear problem, linear analysis is not suitable for practical scenarios. There are various types of batch and recursive algorithms proposed to solve the single target BOT problem $[52,3]$. Next subsection briefs the literature survey of batch processing techniques used in BOT following which the review of recursive algorithms for the same.

\subsubsection{Batch processing techniques}

This type of algorithm processes the batch of measurements for a particular time period to estimate the target state [28]. Nardone et al. [69] have used three technique namely maximum likelihood estimate (MLE), modified-instrumental variable (MIV) and pseudo-linear estimate (PLE) for 2D BOT. The performances of three algorithms are tested for large range-to-baseline scenario. For this scenario, CramerRao bound is derived analytically. The simulation results indicate, for lower effective noise all three algorithms shows similar results and for higher effective noise, PLE shows degraded performance compared to MLE and MIV. In [70], Nardone has proposed the closed form pseudo linear solution to overcome the observability issue caused due to single sensor tracking. It was developed based on the observable parameters which includes bearing, bearing rate and range rate divided by range. Using the observable parameters, normalized polar coordinate state was derived and the results are generated using bearing only measurements. Although this method produces good results for high observability conditions, in case of poor observability the estimates are biased. Later, Kumar et al. [28] also used the modified instrumental variable (MIV) estimate by taking care of missing bearing measurements either randomly or continuously. Performance of the method was evaluated through error bounds. Analogous to [69], Zhang et al. [97] have derived and evaluated the instrumental variable (IV) algorithm based on the covariance matrix. Similar to $[69,70,97]$, Dogancay [18] has proposed weighted instrumental variable (WIV) and compared the performance with MLE, MIV, PLE. He stated that MLE has the disadvantage that, it does not converge to a closed form solution. He also stated that MLE has to be implemented iteratively with the initialization close to the true solution. PLE converges with larger estimation bias. He also added that MIV converges with high computational time. Out of the four techniques used, he proved that the proposed WIV estimator has the closed form solution for the smaller 
bearing noise variance with less computational time and less bias. This method will also diverge with high bearing noise variance [70,52]. Analogous to [18,69,70], Wang et al. [90] have described the pseudo linear least square (PLLS) batch processing method. This method requires no target initialization. The equations for the target state estimation using batch of measurements are derived using standard linear least square method. They proved that, PLLS effectively minimizes the squared norm of the error vector. Huang et al. [37], proposed a bank of batch maximum a posteriori (MAP) estimates to reduce the linearization errors caused due to nonlinear filters and for handling the multihypothesis tracking. The MAP uses the available measurements in each step to compute the state estimate [9, 11]. Each MAP estimator in the bank is initialized by each mode. All the modes are computed analytically by converting the nonlinear cost function into the polynomial form. Thus high probable hypothesis are tracked and thereby increasing the accuracy of the estimation process. Even though MAP batch processing increases the estimate accuracy, these techniques are not preferred due to the larger memory requirement with more computational time for simple scenarios.

\subsubsection{Recursive nonlinear techniques:}

These techniques outperform the batch processing algorithms and produces closed form and unbiased estimates [39]. The common recursive filter used is Kalman filter (KF) for the linear problems. Since BOT is the nonlinear problem, it cannot be used here. The most widely used recursive nonlinear filter is EKF in Cartesian coordinates. The literatures indicate that EKF in Cartesian coordinates may exhibit large error in the target state estimate due to lack of initial range information $[69,45]$. There are various modifications proposed in EKF to overcome this. Other than this new type of Kalman filter namely UKF, CKF are also proposed in the place of EKF. Apart from Kalman filter extensions, SRF and PF are also proposed to estimate the target state in BOT. Following subsection gives the brief review on the proposed extended methods for target state estimation in BOT using the above mentioned nonlinear filters.

\section{A. EKF and its extension}

It is a sub-optimal filter and linearizes the nonlinear measurement model using Taylor series approximation. Aidala et al. [2] have used EKF in modified polar coordinates (EKF-MPC) and the results are compared with EKF-Cart and pseudolinear filter. The authors have assumed the scenario of constant velocity with an arbitrary motion. They showed that, EKF-Cart fails to estimate the target state for long and short range scenario. Similarly, pseudolinear filter produces biased estimates for long range scenario, whereas the EKF-MPC filter shows better performance in all scenarios but they didn't give the analysis for nearly zero or very high bearing rate scenarios. The other robust method used to overcome the drawbacks of above mentioned EKF is Range parameterized-EKF (RPEKF) which was proposed by Peach [74]. This was implemented by dividing the large range uncertainty region into subintervals and the set of weighted EKF is used each with different initial range estimate to obtain the best range estimate $[80,42,96]$. The author has compared the performance of RPEKF with EKF-MPC, EKF-Cart and proved that, RPEKF performs better for nearly zero or very high bearing rate scenarios. Branko et. al. [3], have compared the performance of the particle filter $(\mathrm{PF})$ with EKF-MPC and RPEKF. The authors stated that, PF shows better performance compared to EKF-MPC. Although the RPEKF performance is nearly equivalent to $\mathrm{PF}$ it exhibits larger error initially due to lack of initial target range. The other method which is similar to EKF-MPC is the log polar coordinate (EKF-LPC) and was used by B.L. Scala et. al [83] for BOT. The authors have compared three different tracking algorithms namely EKF-LPC, RPEKF-LPC and Gaussian sum measurement approximation filter. The performance was analyzed using root mean square (RMS) position error, root-time averaged mean square (RTAMS) and number of divergent tracks. The simulation results indicate that, all three filters has similar performance. Another form of EKF is the progressive correction (PC) technique (PC-EKF) and was implemented by Wang et al [90]. They compared the performance of PC-EKF with EKF, RPEKF in MPC (RPEKF-MPC) and PLLS. They showed that, PLLS has better performance than PC-EKF for smaller initial ranges of the target. However at $100 \mathrm{~km}$ EKF, PCEKF and PLLS gives biased estimates of the target. Overall the authors stated that, RPEKF-MPC shows better results compared to other filters irrespective of ranges.

Later, Franken in [20] have explained about the filter initialization of EKF using log spherical coordinates (LSC) and regression based batch estimator. LSC considers both azimuth and elevation measurements and is the $3 \mathrm{D}$ version of LPC used in [83]. Filter initialization using LSC is the one-point initialization technique which consider one measurement pair. Whereas, batch estimator is the multiplepoint initialization technique which consider multiple measurement pairs. The other three estimators considered in this paper are, EKF-Cart initialized with 1. Converted prior 2. Quadratic regression estimator and 3. General non-linear least squares. To validate these techniques, authors have considered two scenarios for target tracking, one is on the passing course and the other is course close to collision. Among all algorithms, EKF-LSC and batch estimator performs better for two scenarios considered. The algorithm which is similar to LSC is modified spherical coordinate (MSC) which is the 3D version of MPC. Mallick et al. [60] have used MSC and LSC for angle only tracking (AOT) in 3D. Authors have presented the new derivation for continuous to discrete EKF-MSC and EKF-LSC filtering algorithms. The derivation for MSC and LSC are presented using first order nonlinear stochastic differential equations and has shown its equivalence with the nearly constant velocity model (NCVM) in Cartesian coordinates. In addition, the authors have presented the new derivation for the predicted covariance which follows the Brownian motion process and it is integrated numerically and jointly to the predicted state estimate to provide better numerical accuracy. Simulations were performed for three different bearing and elevation measurement error standard deviations $(0.001,0.005$ and 0.015 radian) and performance of EKFMSC and EKF-LSC were compared with EKF-Cart. From the results the authors stated that, for high measurement accuracy (0.001 radian) EKF-Cart performs better whereas for medium and low accuracy (0.005 and 0.015 radian) EKF-MSC and EKF-LSC performs better.

The other alternative filtering technique to EKF is the MGEKF. It was initially proposed and derived by [84] and later the derivation of modified gain function was made simple by [22]. Huang et al. [38] has presented the iterated MGEKF, which combines the MGEKF and iteration method. In IMGEKF the new updated state and its 
corresponding covariance are obtained by re-linearizing the measurement function. The other nonlinear filters used are EKF, IEKF, MGEKF. The performances of all the filters are compared with CRLB. The authors stated that IMGEKF has better performance than other filters. However, since this method depends on the updated state for iteration, the disadvantage occurs when the system is unobservable during the initial period of tracking

\section{B. UKF and its extension:}

The other techniques used for constant velocity target state estimation are Sigma point Kalman filter (SPKF) otherwise known as UKF for BOT. It uses the sigma point to linearize the nonlinear dynamic or measurement model, this filter overcomes the divergence problem in EKF [40,59]. Sadhu et al. [82] have made comparison between SPKF, EKF and IEKF. The authors deal with implementation of nonlinear filters for severe nonlinear system with uncertainty in initial conditions. The track lose criterion was taken into account for performance comparison. Simulation results indicate, superiority of SPKF compared to EKF and IEKF. The frequency of track loss in SPKF is $0.014 \%$ whereas for EKF it is $0.28 \%$. The track loss for SPKF can be reduced by changing the $\alpha$. The term $\alpha$ refers to the spread of sigma points. The minimum failure occurs at $\alpha=0.6$. Therefore the authors recommend using SPKF for BOT with acceptable increase in computational time. Analogous to [5,15], Straka et al. [87] has introduced UKF with adaptive scaling parameter. The adaptation was done by means of Maximum likelihood $\left(\mathrm{UKF}^{\mathrm{ML}}\right)$ or Maximum posterior probability $\left(\mathrm{UKF}^{\mathrm{MPP}}\right)$ and has compared with EKF, UKF and SRF for the constant velocity target. The authors have considered three scenarios with different ownship motion patterns. In scenario 1 the ownship follows the straight line motion. The ownship is assumed to take a maneuver in scenario 2. In scenario 3 the ownship follows unit circle centered at origin. From simulation results the authors stated that only in scenario 1, SRF performance was slightly better than UKF's whereas in other scenarios UKF with adaptive scaling parameter performance was good compared to others. More specifically $\mathrm{UKF}^{\mathrm{MPP}}$ achieves better performance.

\section{Cubature Kalman filter (CKF):}

The method of approximating the posterior distribution at each time step with a Gaussian distribution is known as CKF. Cubature is the approximation in a multi-dimensional problem [94]. Analogous to [67], Wu et al. [92] has used CKF along with range parameterized (RP) method. In this paper the sample set of points in the CKF with an orthogonal transformation was designed and used when there is a high degree of nonlinearity in the measurement function. The authors refer to these points as orthogonal simplex cubature points (OSCPs). This paper also used the range parameterized (RP) method with different initial estimates, which deals with fuzzy initial estimation problem. The RP tracker has demerits in terms of computational complexity, due to the usage of series of sub-filters. Authors tried to reduce the computational cost by setting the threshold and removal of unstable sub-filters. But still the authors stated that the proposed RPOSCKF has high computational complexity but performs better than other conventional nonlinear filters used.

\section{Shifted Rayleigh filter (SRF) and its extension:}

It is a moment matching algorithm used for BOT to calculate the exact conditional mean and covariance for the given measurement [5]. Sanjeev et.al [5] have used SRF for Maximum bearing rate (MBR) scenarios. The authors have investigated the performance of SRF with EKF, UKF and PF for the above mentioned scenario. SRF uses the directional cosines of bearing measurement and augmented to obtain the exact conditional mean and covariance. From the simulation results, it is stated that EKF and UKF shows divergent tracks for low MBR whereas SRF and PF does not shows divergent tracks even with increased MBR. Hence SRF achieves performance similar to PF. Ozelci et al. [15] have used SRF for target tracking from noisy measurements in the presence of clutter, and was named as SRF for 3D bearing measurement with clutter (SRF3C). The authors have compared performance of proposed SRF3C with EKF, RPEKF, UKF and Particle filter with local EKF linearization (EKPF), considering high bearing rate scenario. The simulation results indicate EKF, UKF and RPEKF perform poor whereas EKPF has the comparable performance to that of SRF3C but has higher computational time nearly 4 orders of magnitude compared to SRF3C. Hence it is stated that, the proposed SRF3C performs better in case of poor target initialization, high probability of clutter and high bearing rate scenarios.

\section{E. Particle filter and its extension:}

It is the recursive Monte Carlo method which represents the posterior density of target $[62,66]$. Gordon et al. [25] have described the sampling based method and auxiliary PF for BOT problem. The performances were compared with EKF. From results, the best performance is achieved by auxiliary PF whereas EKF estimate diverges. Later, Karlsson et al. [41] have used PF in MSC and Cartesian. The comparison was made between RPEKF and PF in Cartesian and MSC. The results indicate the good performance of PF in both MSC and Cartesian with more computational time than RPEKF. Mallick et al. [61] have used UKF and PF in MSC. This work is an improved version of his work in [60] reviewed in subsection (2.1.2.A). In this paper an improved filter initialization algorithm was discussed. The authors have used EKF, UKF and Bootstrap particle filter (BPF) in Cartesian and MSC. The simulation results indicate for high measurement accuracy, EKF and UKF in MSC performs marginally better than EKF and UKF in Cartesian. For low measurement accuracy best performance was achieved by EKF-MSC and UKF-MSC compared to others. Whereas $\mathrm{BPF}$ shows severe computational complexity than others and performance was not better than EKF-MSC and UKF-MSC. The authors stated that, the poor performance of BPF can be increased by adding particles but this will further increase computational time. Similar to [60,61], Gupta et al. [29] considered PFF along with deterministic Ensemble Kalman filter (DEnKF) and stochastic Ensemble Kalman filter (sEnKF) in Cartesian and MSC and compared the results with EKF, UKF, BPF in Cartesian and MSC. In the case of low measurement accuracy, the simulation results indicates better performance for PFF in Cartesian and MSC whereas similar performance was achieved by EKF, UKF and DEnKF only in MSC and not in Cartesian. Also PFF performs better with less number of particles compared to particles used in BPF.

Ristic et. al [79] have discussed the improved and modified form of PF called Bernoulli PF and is used for ownship motion control under the consideration of false alarm and missed detection. Problem is to identify the appearance and disappearance of target during ownship motion. To solve this problem, Bernoulli filter was used. 
Since Bernoulli filter does not form a closed-form solution it is implemented based on the particle filter with diffuse prior. Further observer motion was controlled using the output of Bernoulli PF. The simulation was done with two different average number of false detections $\lambda=0.5$ and $\lambda=5$. The results indicate that for $\lambda=0.5$, the RMS error is very close to theoretical bound and for $\lambda=5$ the estimation error increases. Overall the performance of Bernoulli PF shows better performance in track maintenance. Analogous to [79], Morelande [66] has developed marginalized particle filter (MPF) for 2D BOT. This paper considers the improvement of PF known as marginalization technique. This can be done by replacing the Monte Carlo approximation with analytical computation. In this paper Euler approximation is used for dynamical equation and thus only three elements of the state vector needs to be sampled. The proposed MPF in MPC (MPF-MPC) was compared with MPF in Cartesian (MPF-C) and Bootstrap filter in Cartesian (BF-C) and MPC (BFMPC). From the simulation results, MPF-MPC shows best performance compared to others with one $10^{\text {th }}$ of sample size but with greater computational time.

\section{F. Kernel based filter $(\mathrm{KBF})$ :}

This method is mainly used for signal propagation time delay. Yunfei et al [27] have used online parameter estimation (OPE) method embedded into a nonlinear filter for tracking moving target. Along with OPE the authors have used improved range parameterized EKF (IRP-EKF) in which adaptive weight adjustment is introduced. Further instead of EKF, they used Kernel-based filter (KBF) and regularized particle filter (RPF) to improve the filter performance. The proposed OPE-IRP-KBF based estimation is compared with OPE-RPEKF and OPE-regularized particle filter (OPE-RPF). The authors stated that proposed method runs four parallel $\mathrm{KBF}$ during the initial stage and remove the low-weight sub-intervals and produces better fusion result. During the normal tracking stage it produces high estimation accuracy, compared to other methods but the computational time is higher. Thus the authors stated that, the proposed technique is better with an acceptable high computational time.

\subsubsection{Smoothing techniques:}

The process of Smoothing is different from filtering. Smoothing not only process measurements up to the particular time but also considered the delayed measurements. Hence smoothing process is better optimization than filtering [1]. The process of smoothing is applied to BOT and is discussed by few authors. In analogy to [38], Qian et al. [73] has proposed a smoothing MGEKF (sMGEKF) based on Rauch-Tung-Stribel (RTS) smoothing. The authors stated that, RTS algorithm is a fixed interval smoothing and it has two steps forward filtering and backward propagation process. The forward filtering employs the usual filtering algorithm and backward processing propagates the statistics of the filter backward in time and obtains the smoothed states. The nonlinear filters used in this paper are, EKF, IEKF, smoothing EKF (sEKF), MGEKF and sMGEKF. From simulation results, the authors concluded that sMGEKF performs better during the initial period when the target is not observable and also reduces the estimation error. Similar to [73], Meiqin et al. [67] have combined the RTS smoother and Cubature Kalman filter and presented the Cubature Rauch-Tung-Striebel (CRTS) smoother. Based on this the authors has proposed the new technique cubature Rauch-Tung-Striebel (CRTS)-U. This was performed to check the optimal ownship maneuver using the determinant of covariance matrix and the trace of it. CKF, CRTS and CRTS-U algorithms were compared and proved. CRTS-U has superior performance with less RMS error.

\subsection{Satellite applications:}

BOT was also used in satellite applications and is explained by $\mathrm{Li}$ et al. [49]. The authors have explained about the new passive EKF-Cart tracking method using bearing only measurements for satellite to satellite tracking applications. The bearing measurements from J2000 ECI frame is used for state transfer matrix and Jacobian matrix calculation. Even with the larger initial errors, with EKF-Cart the results are converging. This can be used to passively track low earth circular orbit satellite by a high earth orbit satellite. Li et al. extended the same work in [50] by considering the observability problem between the satellite to satellite passive tracking. They explained and proved mathematically the necessary and sufficient condition for satellite tracking. Qiang et al. [72] have used MSC-EKF for satellite application and verified through simulation that, it produces unbiased estimation with fast convergence compared to EKF-Cart. The Table 1 gives the brief overview of the techniques and its performance analysis used by different authors for category 1 .

Table 1. Different algorithms used for single sensor and single target with constant velocity and their performance analysis

\begin{tabular}{|c|c|c|}
\hline $\begin{array}{l}\text { Authors, reference and } \\
\text { year }\end{array}$ & Innovation & Performance analysis \\
\hline $\begin{array}{l}\text { V.J. Aidala et. al } \\
\text { [2], } 1983\end{array}$ & EKF using modified polar coordinate (EKF-MPC). & $\begin{array}{l}\text { EKF-MPC performs better and avoids filter } \\
\text { instability compared to pseudolinear filter, and } \\
\text { EKF-Cart. }\end{array}$ \\
\hline $\begin{array}{l}\text { P.J. Galkowski et.al } \\
\text { [22], } 1991\end{array}$ & Derived a new form of gain for MGEKF. & $\begin{array}{l}\text { Performance of MGEKF was better than standard } \\
\text { EKF. }\end{array}$ \\
\hline nan & EKF-MPC formulated using Line-of sight (LOS) algebra & EKF-MPC performs better than standard EKF. \\
\hline $\begin{array}{l}\text { N. Peach } \\
{[74], 1995}\end{array}$ & Range parameterized (RP) tracker using EKF-Cart is used. & $\begin{array}{l}\text { RP tracker performs better compared to EKF-MPC } \\
\text { and EKF-Cart. }\end{array}$ \\
\hline $\begin{array}{l}\text { N. Gordon et.al [25], } \\
1998\end{array}$ & Sampling-Importance resampling (SIR) filter, Auxillary SIR filter. & $\begin{array}{l}\text { SIR performs better compared to EKF and Auxillary } \\
\text { SIR filter. }\end{array}$ \\
\hline $\begin{array}{l}\text { T.R. Kronhamn [42], } \\
1998\end{array}$ & $\begin{array}{l}\text { Adaptive ownship motion using Multihypothesis Cartesian } \\
\text { Kalman filter (MHCKF) }\end{array}$ & $\begin{array}{l}\text { The adaptive ownship motion using MHCKF shows } \\
\text { good results compared to fixed ownship motion. }\end{array}$ \\
\hline $\begin{array}{l}\text { S. Arulampalam et. al } \\
{[3], 2000}\end{array}$ & $\begin{array}{l}\text { Comparison of various nonlinear filters for angle only } \\
\text { measurements }\end{array}$ & $\begin{array}{l}\text { better performance compared to RPEKF } \\
\text { IPC }\end{array}$ \\
\hline $\begin{array}{l}\text { R. Karlsson et.al } \\
\text { [41], } 2001\end{array}$ & PF-MSC and RPEKF-MSC. & $\begin{array}{l}\text { PF-MSC performs better with high computational } \\
\text { time compared to PF-Cart and RPEKF-Cart. }\end{array}$ \\
\hline $\begin{array}{l}\text { S. Sadhu et.al } \\
{[82], 2006}\end{array}$ & Sigma point Kalman filter (SPKF) & $\begin{array}{l}\text { SPKF performs better than EKF, iterated EKF } \\
\text { (IEKF). }\end{array}$ \\
\hline S.Arulampalam et.al & Shifted Rayleigh filter (SRF) & SRF performs better than EKF, UKF and PF \\
\hline
\end{tabular}


[5], 2007

Y.Guo et.al

[27], 2008

B.L. Scala et.al

[83], 2008

X. Wang et. al

[90], 2009

O. Straka et.al

[87], 2011

M. Mallick et.al

[60], 2011

B. Ristic et.al

[79], 2012

A. Can Ozelci et.al

[15], 2012

M.R. Morelande

[66], 2013

P.H.Leong et.al

[57], 2013

Z.Qian et.al

[73], 2014

L.Meiqin et.al

[67], 2015

D.Franken

[20], 2015

Y.Huang et al

[38], 2015

S.D. Gupta et al

[29], 2015

H.Wu et.al

[92], 2016

Q.Zhang et al

[98], 2016
Online parameter estimation-Improved range parameterizedKernel based filter (OPE-IRP-KBF).

\section{EKF-LPC and RPEKF-LPC}

1. Pseudo linear least square (PLLS) filter,

2. RPEKF-MPC,

3. Progressive correction EKF (PC-EKF).

UKF with adaptive scaling parameter

Continuous-discrete EKF using MSC and LSC

Bernoulli PF was used in maintaining tracks in difficult scenarios.

SRF for 3D bearing measurements with clutter (SRF3C).

Marginalised Particle filter (MPF) and Bootstrap filter (BF) in MPC and Cartesian coordinates.

Gaussian-sum Cubature Kalman filter (GSCKF), RP Cubature Kalman filter (RPCKF).

1. Smoothing MGEKF (sMGEKF)

2. Rauch-Tung-Striebel (RTS) smoothing.

Cubature RTS-U (CRTS-U)

Regression-based batch estimator for track initialization.

\section{IMGEKF}

1. Cartesian PFF (CPFF),

2. Cartesian deterministic Ensemble Kalman filter (CDEnKF),

3. Cartesian stochastic EnKF (CSEnKF),

4. $\mathrm{CPFF}$ (local)

5. SEnKF-MSC,

6. EnKF-MSC.

1. RP orthogonal simplex CKF (RPOSCKF)

2. RPOSCKF-I improved range parameterized strategy (IRP)

3. Simplex CKF (SCKF)

4. Orthogonal simplex cubature points (OSCPs).

Smoothing EKF (sEKF) and smoothing MGEKF (sMGEKF).
The proposed OPE-IRP-KBF performs better than Online parameter estimation RPEKF (OPERPEKF), OPE regularized PF (OPE-RPF), OPE Kernel-based filter (KBF)

EKF-LPC and RPEKF-LPC performs better than Gaussian sum measurement approximation filter. The better performance was achieved by RPEKFMPC compared to PC-EKF, EKF and PLLS.

UKF with adaptive scaling parameter performs better than EKF, UKF, SRF.

CEKF performs better for high measurement accuracy and in case of medium measurement accuracy EKF-MSC and EKF-LSC performs better. Bernoulli PF performs better.

SRF3C performs better compared to EKF, UKF, RPEKF, sampling-importance-resampling particle filter with local EKF linearization (EKPF).

$\mathrm{MPF}$ in MPC has better performance than BF in MPC.

The analysis of performance indicates GSCKF has performance similar to $\mathrm{PF}$ and better performance than EKF, PF, UKF, CKF.

SMGEKF performs better compared to MGEKF,EKF and IEKF.

The better performance was achieved by CRTS-U compared to Cubature RTS (CRTS) smoother and CKF.

The batch estimator using regression based multipoint initialization performs better than EKFLSC, CEKF and EKF with prior, nonlinear least square.

IMGEKF performs better than MGEKF, EKF,

IEKF.

The best performance was achieved for EKF, UKF and DEnKF in MSC and PFF than EKF-Cart, UKFCart, BPF-Cart, CPFF(local), EKF-MSC, UKFMSC, BPF-MSC, SEnKF-MSC, EnKF-MSC, PFFMSC.

RPOSCKF algorithm shows better performance compared to other techniques.

sEKF and SMGEKF performs better compared to EKF.

3. Review of techniques for category 2

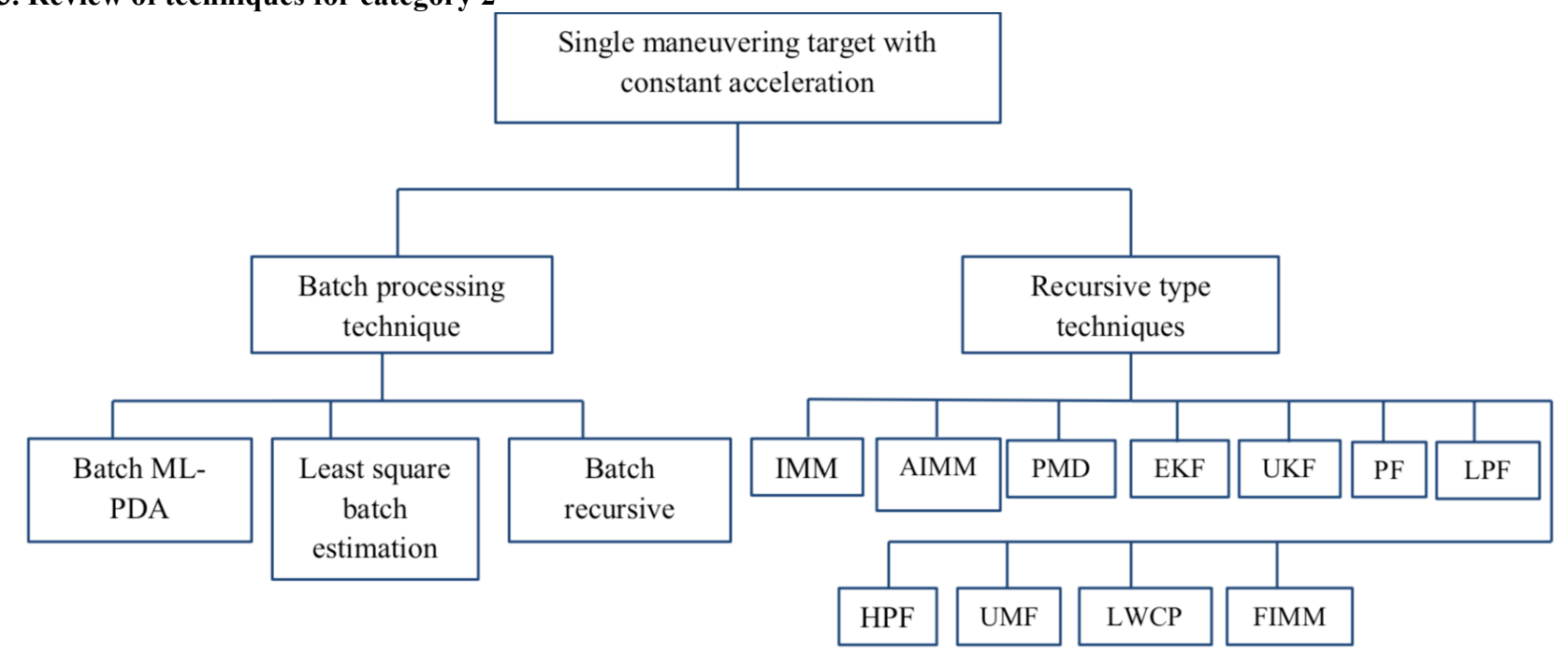

Fig. 3. Different techniques involved for maneuvering target tracking 
- $\quad$ IMM - Interactive multiple model

- AIMM - Adaptive interactive multiple model

- $\quad$ PMD - probability mass diffusion filter

- $\quad$ LPF - learning particle filter

- HPF - hierarchical particle filter

- $\quad$ UMF - unscented mixture filter

- $\quad$ LWCP - Liu and West change point filter

- $\quad$ FIMM - Fuzzy-logic-based IMM

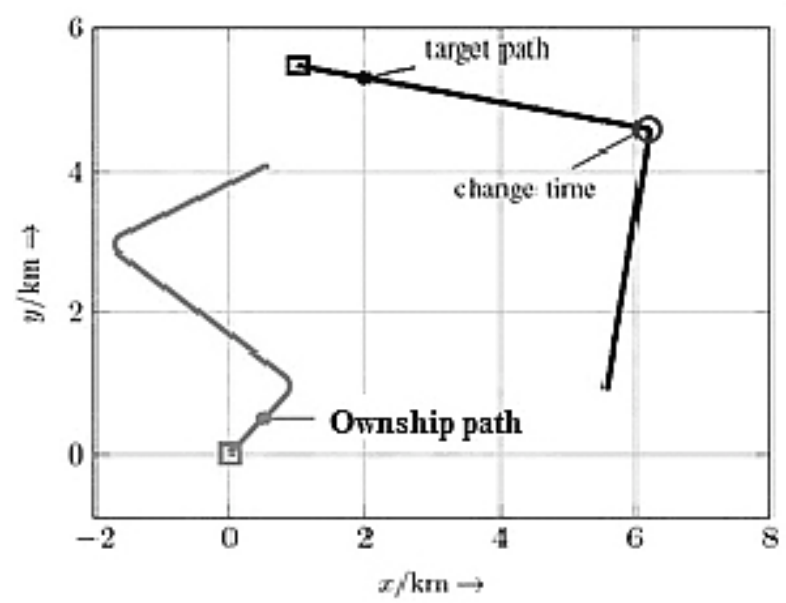

(a)

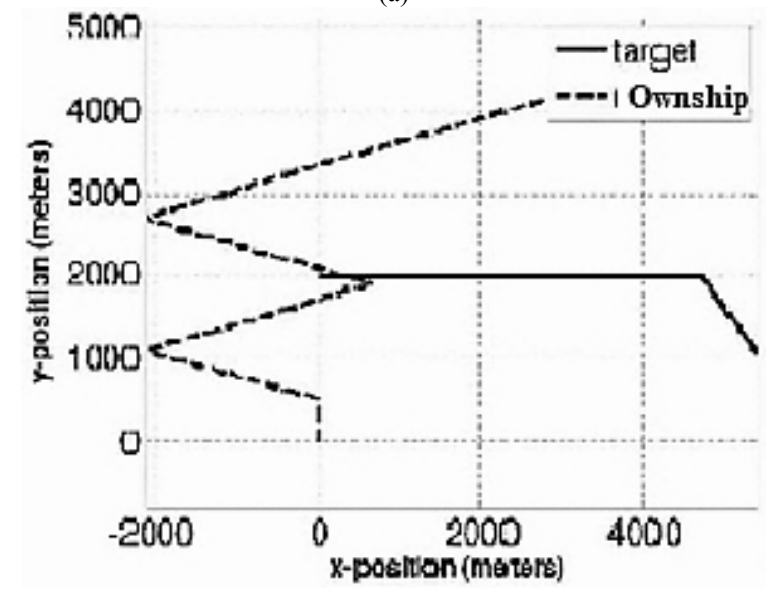

(b)

Fig.4. Different ownship and target maneuvering patterns for single sensor target tracking when the target moves at constant acceleration as shown in [a], [b] courtesy [36], [102].

This section deals with techniques used to estimate the state of the maneuvering target in BOT. Fig. 3 gives the block diagram of different techniques involved for maneuvering target and Fig. 4 shows the different maneuvering patterns of target and ownship. The number of literature related to maneuvering target was less. The maneuvering target corrupts range estimation and produces incorrect state estimates $[7,55]$. There are various techniques involved in estimating the state of maneuvering targets in air space application and underwater application depending upon the nature of maneuvers and are explained briefly in the following sections.

\subsection{Air space applications}

\subsubsection{Batch processing techniques:}

Batch processing can also be used for this category. Allen et al. [7] have presented a tracking filter for BOT. In this paper two types of maneuver detection methods are presented namely a. conventional method and $b$. batch processing method. The conventional method works by comparing the variance of normalized squared residuals of angle and angle rate in azimuth and elevation with chi-squared threshold [8]. The authors stated that this method is useful for detecting low-level maneuvers, but it was not effective than that of batch estimation. In batch processing technique, sum of squares (SOS) of measurement residuals is calculated and was approximated to follow a chi-square distribution $\chi_{M}^{2}(\mathrm{M}$ is the number of degrees of freedom). The sliding windows of $\mathrm{N}$ observations are processed to determine whether, target maneuver occurred within the window. The maneuver was detected by comparing SOS with the threshold from $\chi_{M}^{2}$ distribution. If SOS results in a poor fit, then system declares that detection of target maneuver is done. Later, Liu et al. [52] have presented the novel hybrid estimator for $2 \mathrm{D}$ maneuvering target in BOT. The hybrid estimator combines two algorithms, instrumental variable pseudolinear estimator (IV-PL) and pseudolinear Kalman filter (PLKF) for detection of target maneuver. Initially, PLKF is used for initialization of hybrid estimator and it is checked for convergence of target maneuver. If the target dynamics is not converging IV-PL estimator is used for estimation of the target maneuver. From the simulation results the combination of two estimators shows the superiority of the hybrid algorithm. Later, Kiruba et al [45] has stated that, batch processing type techniques cannot handle target maneuvers and hence a combination of batch and recursive estimator is proposed for maneuvering target in clutter environment. In this paper, the interacting multiple model probabilistic data association filter with amplitude information (IMMPDAFAI) is proposed for maneuvering targets detection in clutter. Initially ML-PDA batch method is implemented for accurate initialization of IMMMPDAFAI to provide reliable track maintenance. The authors have proved that, the proposed algorithm is effective even with $8 \mathrm{~dB}$ SNR.

\subsubsection{Recursive nonlinear techniques:}

There are number of techniques involved for solving nonlinear measurement equation, but most of the methods produces filter divergence and biased estimates for highly maneuvering scenarios. Brehard et al. [12] have used the Best fitting Gaussian (BFG) distribution for the computation of posterior Cramer-Rao bound (PCRB) for the maneuvering target in BOT. The maneuvering target was modeled by Jump Markov linear model. Since measurement model is nonlinear for BOT, the closed form solution cannot be obtained $[34,89,100]$. Hence, to obtain the closed form solution BFG model associated with BOT was calculated using Log polar coordinate (LPC) system and was tested for maneuvering target to obtain the closed form expression for PCRB. Xu. et al. [93] has proposed an easy-to-implement maneuver detection method for detecting the maneuvering target in BOT. The authors have also used the fuzzy-neuralnetwork (FNN) for maneuver detection. Two scenarios are considered one is, obtaining the exact CRLB curves based on known a priori maneuvering time and second is investigation of CRLB for the proposed method and FNN method. The performance was compared with CRLB obtained through a proposed maneuver detection method and FNN method with respect to true CRLB. The authors stated that, from simulation results the proposed maneuver detection method performs better than FNN maneuver detection. 


\section{A. Particle filter and its extension:}

This sub-section deals with particle filter and its extended versions used for tracking the maneuvering target in BOT. Even though particle filter has high computational time as stated in sub-section 2.1.2.E. particle filters are mostly used for highly maneuvering targets especially in BOT. Brehard et al. [14] have proposed hierarchical particle filter (HPF) for highly maneuvering target scenarios in BOT. In this paper the performance of $\mathrm{PF}$ was analyzed using Log polar coordinate (LPC). The authors stated that, traditional PF diverges at some point due to unobservability of radial distance between the target and ownship also the speed bounds of the target. To overcome the problem of filter divergence in PF, HPF is introduced and track initialization was done using a simple Monte-Carlo Markov chain (MCMC) method. The comparison was done between the bootstrap PF and HPF with two initial priors. The simulation results for prior 1 indicate that, during the initial stage performance of both filters are same. After some time, bootstrap filter diverges but HPF shows better performance. Similarly for prior 2 during initial stage both filters suffer from bias due to initial prior. The filter divergence occurs for bootstrap filter whereas HPF performs better. It has been confirmed that for more difficult scenario, the proposed method outperforms classical bootstrap filter. Similar to method proposed earlier by Brehard et al. [14], Horst et al. [36] have used marginalized particle filter (MPF) for target state estimation in BOT. The authors initially consider bearing-only measurement (BOTMA) and later they proposed to consider bearing rate measurements (BRTMA) along with bearing measurement. The proposed bearing measurements are used in MPF not only for target state estimation but also detecting the change in the maneuver of the target. In this paper, target maneuver was detected using chi-square hypothesis test with window length of 10 and threshold of 18.3 with confidence level of $95 \%$. The simulation results indicate the increase in estimation accuracy as well as identifying the maneuver change detection. Nemeth et al. [71] have proposed learning particle filter (LPF) to detect the target maneuver in unknown period of time. This paper considers joint state estimation of maneuvering targets with unknown model parameters. The proposed LPF is used for state estimation and unknown model parameters through online. The other successful online static parameter estimation used in this paper is The Liu and west filter [91] and LPF [17]. In this paper it is shown that these two methods can be further extended to estimate piecewise time varying parameters by considering change point analysis. The comparison was made between Liu and West change point (LWCP) filter and IMM filter for two scenarios. Scenario 1 considers unknown turn rate parameter $\omega$ and scenario 2 considers unknown system noise variance $\tau^{2}$ along with unknown $\omega$ as stated in scenario 1. IMM filter is implemented with the mixture of UKF filters and the results are compared with LWCP filter which is implemented with 10,000 particles. Since LWCP filter is able to learn parameters online, simulation results shows better performance during target maneuver than IMM filter. The comparative results indicate better estimation for LWCP filter than IMM.

\section{B. Shifted Rayleigh filter and its extension:}

According to the literature when target is in a maneuvering condition, target state estimation can be achieved better when improved SRF is used instead of SRF. Clark et al. [16] have proposed shifted Rayleigh mixture filter (SRMF) for BOT of maneuvering targets. The authors have considered the problem of maneuvering target by taking clutter in to account for each maneuver mode. For this problem, the conditional density of the target state for available measurements is assumed to follow the Gaussian mixture of probability densities. The authors stated that the number of component in the mixture grows in time hence, SRMF based on jump Markov linear systems was used to obtain the exact solution which depends only on the first and second moment of conditional density of the state. The performance comparison was done between SRMF with PF and unscented mixture filter (UMF). The proposed method achieves accuracy as that of PF with less computational complexity compared to UMF and SRF. The authors stated that, proposed method was also effective for multiple sensors.

\section{Interactive Multiple model (IMM)and its extension:}

This method uses the multiple models to handle the target maneuvers in BOT. Kronhamn [43] has proposed an Adaptive-IMM Multiple-Range-Models (AIMM-MRM) for estimating the range of maneuvering target. The proposed algorithm is an extension of IMM technique with adaptive transition probabilities determined based on maneuver detection of the target. The authors have considered four different estimators, non-maneuvering MRM (nom-MRM), maneuvering-MRM (man-MRM), IMM-MRM and AIMMMRM for both maneuvering and non-maneuvering case. From the simulation results it is stated that, AIMM-MRM method performs better and is suitable for range estimation with non-maneuvering and maneuvering models. Further Kronhamn [44] has proposed another new MRM using probability mass diffusion filter (PMD). This PMD is used to find the probability of interaction between maneuvering and non-maneuvering models was according to PMD principle. The models considered for simulation are MRMPMD, MRM-IMM and MRM of non-maneuver model (nom-MRM). From the results, author stated that for maneuvering targets IMM performs better than PMD. For non-maneuvering targets, the performance of PMD is better than IMM and identical to nom-MRM. Overall, the performance of IMM is good for maneuvering target.

\subsection{Underwater applications:}

In underwater target tracking scenarios, the noise in the measurements are very high. The speed and turning rate of the ownship are low as compared with the air target tracking scenarios. To overcome these difficulties, the widely used nonlinear filter for underwater target tracking is MGEKF [46]. Koteswara Rao [47] has used MGEKF proposed in [22] for maneuvering target tracking in underwater scenarios. This algorithm detects target maneuver by assuming the observation follows zero mean chi-square distribution in sliding window format [46] by considering the known initial parameters like target range, speed and azimuth angle. The size of the window is assumed to be five for highly noisy underwater scenarios. The threshold value of 30 was chosen to avoid false target maneuver detection. If normalized squared innovations exceed the threshold target maneuver is detected. When the target was not maneuvering the process noise is assumed to be 0.01 and during target maneuver the process noise was assumed to be 10 and it was chosen based on the results from the number of geometries from Monte Carlo simulation. This method was similar to conventional maneuver detection by [7]. The disadvantage 
of the algorithm is that, it produces poor estimates for the scenarios of target moving away from the ownship. Similar to method explained in [47], Koteswara rao [48] have presented improved method of tracking maneuvering target for highly maneuvering scenarios using UKF. The authors stated that, for highly maneuvering scenarios traditional EKF and MGEKF fail because of filter divergence. UKF detects target maneuver based on zero mean chi-square distribution in sliding window format. The target maneuver is detected when the normalized squared innovations exceed the threshold. The concept of maneuver detection using threshold was already explained in [46, 47]. When the target is not maneuvering, the normalized squared innovation is less than the threshold. The results were analyzed using Monte Carlo simulation and performance of UKF is better for highly maneuvering scenarios. The Table 2 gives the brief overview of the techniques used and its performance analysis used by different authors for category 2 .

Table 2.Algorithms used and its performance analysis for single sensor and single target with constant acceleration

\begin{tabular}{|c|c|c|}
\hline $\begin{array}{l}\text { Authors, reference } \\
\text { and Year }\end{array}$ & Innovation & Performance analysis \\
\hline $\begin{array}{l}\text { R. R. Allen et al } \\
{[7], 1991}\end{array}$ & $\begin{array}{l}\text { Least-squares batch estimation technique for maneuver } \\
\text { detection }\end{array}$ & $\begin{array}{l}\text { Least-squares batch estimation technique performs } \\
\text { better. }\end{array}$ \\
\hline $\begin{array}{l}\text { J.P.Le Cadre et al } \\
{[55], 1998}\end{array}$ & $\begin{array}{l}\text { 1. Hidden Markov model (HMM), } \\
\text { 2. classical dynamic programming (DP), } \\
\text { 3. Partially observable Markov decision process (POMDP) }\end{array}$ & $\begin{array}{l}\text { Simulation results indicate, DP algorithm performs } \\
\text { better. }\end{array}$ \\
\hline $\begin{array}{l}\text { S.Koteswara Rao } \\
{[46], 1999}\end{array}$ & MGEKF for underwater applications & $\begin{array}{l}\text { The simpler version of modified function is used and } \\
\text { the performance was found to be better. }\end{array}$ \\
\hline $\begin{array}{l}\text { T. Kirubarajan et al [45], } \\
2001\end{array}$ & $\begin{array}{l}\text { 1. Batch Maximum likelihood-probabilistic data association } \\
\text { (ML-PDA) for filter initialization. } \\
\text { 2. IMM PDA with amplitude information (IMMPDAFAI) }\end{array}$ & $\begin{array}{l}\text { The method of recursive IMMPDAFAI using } \\
\text { coordinate turn models performs better in maintaining } \\
\text { tracks and to enhance the observability. }\end{array}$ \\
\hline $\begin{array}{l}\text { T.R.Kronhamn } \\
{[43], 2002}\end{array}$ & Adaptive IMM Multiple-Range-models (AIMM-MRM). & $\begin{array}{l}\text { AIMM-MRM technique performs better compared to } \\
\text { non maneuvering MRM (nom-MRM), maneuvering } \\
\text { MRM (man-MRM), IMM-MRM. }\end{array}$ \\
\hline $\begin{array}{l}\text { T. Brehard et al } \\
{[12], 2006}\end{array}$ & Best-fitting Gaussian (BFG) model in LPC & $\begin{array}{l}\text { The closed form PCRB was derived using BFG model } \\
\text { in LPC system and found to give better results. }\end{array}$ \\
\hline $\begin{array}{l}\text { F. Bavencoff et al } \\
{[101], 2006}\end{array}$ & $\begin{array}{l}\text { 1. Monte Carlo Markov chain (MCMC) } \\
\text { 2. Highest probability density (HPD) intervals method, } \\
\text { 3. Hit-and-Run algorithm }\end{array}$ & $\begin{array}{l}\text { MCMC algorithm shows better performance } \\
\text { compared to others. }\end{array}$ \\
\hline T.Kronhamn [44], 2007 & $\begin{array}{l}\text { 1. multiple range models and probability Mass diffusion } \\
\text { (MRM-PMD) } \\
\text { 2. MRM-IMM }\end{array}$ & $\begin{array}{l}\text { PMD performs better for non-maneuvering targets and } \\
\text { IMM performs better for maneuvering targets. }\end{array}$ \\
\hline $\begin{array}{l}\text { B.Xu et al } \\
{[93], 2007}\end{array}$ & Proposed Easy-to-implement maneuver detection method. & $\begin{array}{l}\text { Proposed maneuver detection performs better than } \\
\text { Fuzzy neural network (FNN) }\end{array}$ \\
\hline $\begin{array}{l}\text { J.M.C.Clark et al } \\
{[16], 2007}\end{array}$ & $\begin{array}{l}\text { 1. Shifted Rayleigh mixture filter (SRMF) 2. Unscented } \\
\text { mixturefilter (UMF). }\end{array}$ & $\begin{array}{l}\text { SRMF performs better interms of computational time } \\
\text { and achieves accuracy similar to PF. }\end{array}$ \\
\hline T. Brehard et al [14], 2007 & $\begin{array}{l}\text { 1. Hierarchical particle filter }(\mathrm{HPF}) \text {, } \\
\text { 2. Bootstrap filter }(\mathrm{BF})\end{array}$ & HPF using LPC performs better. \\
\hline $\begin{array}{l}\text { S. Koteswara Rao et al } \\
{[48], 2008}\end{array}$ & UKF-MSC for underwater application & $\begin{array}{l}\text { The performance of UKF-MSC was found to give } \\
\text { better results. }\end{array}$ \\
\hline $\begin{array}{l}\text { J.Horst et al } \\
{[36], 2011}\end{array}$ & $\begin{array}{l}\text { Marginalized particle filter (MPF) using bearing and } \\
\text { bearing rate measurements }\end{array}$ & MPF performs better. \\
\hline C. Nemeth et al [71], 2012 & $\begin{array}{l}\text { 1.Particle learning filter (PLF) } \\
\text { 2.Liu and west filter } \\
\text { 3. Particle learning change point filter }\end{array}$ & $\begin{array}{l}\text { PLF achieves better performance compared to IMM } \\
\text { filter. }\end{array}$ \\
\hline Y.J. Zhang et al [97], 2013 & $\begin{array}{l}\text { 1. Shiryayev sequential probability ratio test (SSPRT), } \\
\text { 2. Proposed acceleration estimation (AE) maneuver detector } \\
\text { 3. proposed bearing based detector (BD detector) }\end{array}$ & $\begin{array}{l}\text { The AE detector performs better than } \\
\text { BD detector. }\end{array}$ \\
\hline $\begin{array}{l}\text { E. Tom Northardt et al } \\
{[102], 2014}\end{array}$ & $\begin{array}{l}\text { 1. Expected likelihood maneuver detector (ELMD), } \\
\text { 2. Koteswara maneuver detector, } \\
\text { 3. Measurement residual (MR) maneuver detector }\end{array}$ & $\begin{array}{l}\text { ELMD maneuver detector technique shows better } \\
\text { compared to MR and Koteswara maneuver detectors. }\end{array}$ \\
\hline $\begin{array}{l}\text { H. Seung Son et al } \\
{[103], 2014}\end{array}$ & $\begin{array}{l}\text { 1.Fuzzy C-means clustering (FCM), } \\
\text { 2.Fuzzy-logic-based IMM (FIMM), } \\
\text { 3.proposed complementary compensation method }\end{array}$ & $\begin{array}{l}\text { The proposed complementary compensation performs } \\
\text { better. }\end{array}$ \\
\hline $\begin{array}{l}\text { A.K. Mohammadiyan et al } \\
{[65], 2016}\end{array}$ & Proposed adaptive IMM-PF & $\begin{array}{l}\text { The proposed adaptive IMM-PF algorithm shows } \\
\text { better performance than IMM-PF. }\end{array}$ \\
\hline $\begin{array}{l}\text { L. Qun Li et al } \\
\text { [104], } 2016\end{array}$ & $\begin{array}{l}\text { 1.Auxiliary truncated Particle filtering with prior (ATPF) } \\
\text { 2.modified prior probability density function (PDF) } \\
\text { 3.IMM Rao-Blackwellized Particle filter (IMMRBPF) }\end{array}$ & $\begin{array}{l}\text { ATPF algorithm performs better compared to PF } \\
\text { algorithms, EKF, UKF, modified truncated UKF } \\
\text { (MTUKF), PF-EKF, UPF, and shows improved }\end{array}$ \\
\hline
\end{tabular}


4. Review of techniques for category 3 :

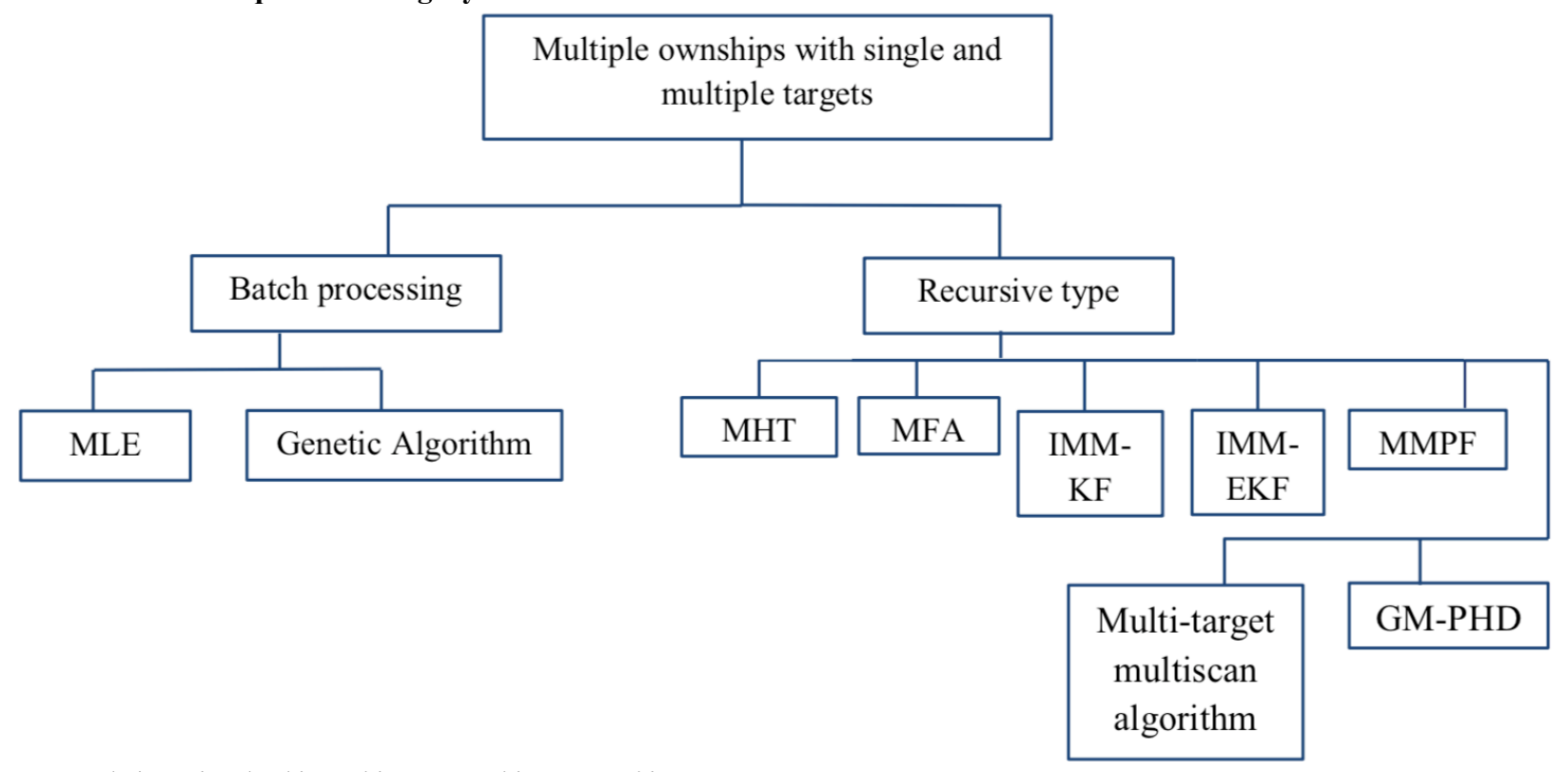

Fig. 5. Techniques involved in Multi-sensor multi target tracking

- $\quad$ MHT - $\quad$ Multiple hypothesis tracker

- MFA - Multiframe assignment algorithm

- $\quad$ MMPF - Multi-mode particle filter

- MLE - Maximum likelihood estimate

- GM-PHD - Gaussian mixture probability density hypothesis

This category review on single and multiple targets tracking with single and multiple sensors. Fig. 5 gives the overview of the techniques involved in multi-sensor multitarget tracking. Observability is not a major problem for this scenario since multiple sensors are involved [88]. Major challenges are measurement origin uncertainty, lack of knowledge about the number of targets in the surveillance region, track maintenance, clutter detection, false alarm, missed detection and appearance of ghost targets. These constrained have to be taken into account when we track a particular target by estimating its state at every instance of time. These problems can be solved by considering the various data association techniques to identify the similar information pertaining to a particular target and fused using fusion techniques to obtain the optimized target sate.

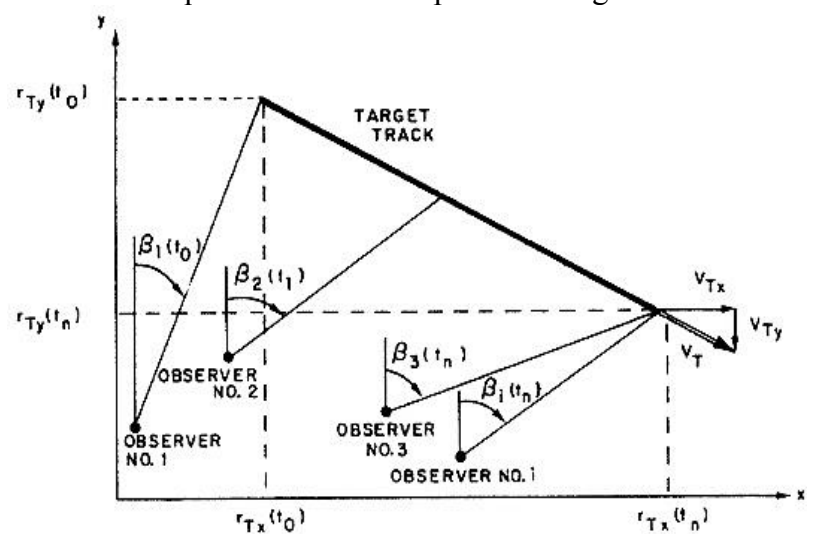

(a)

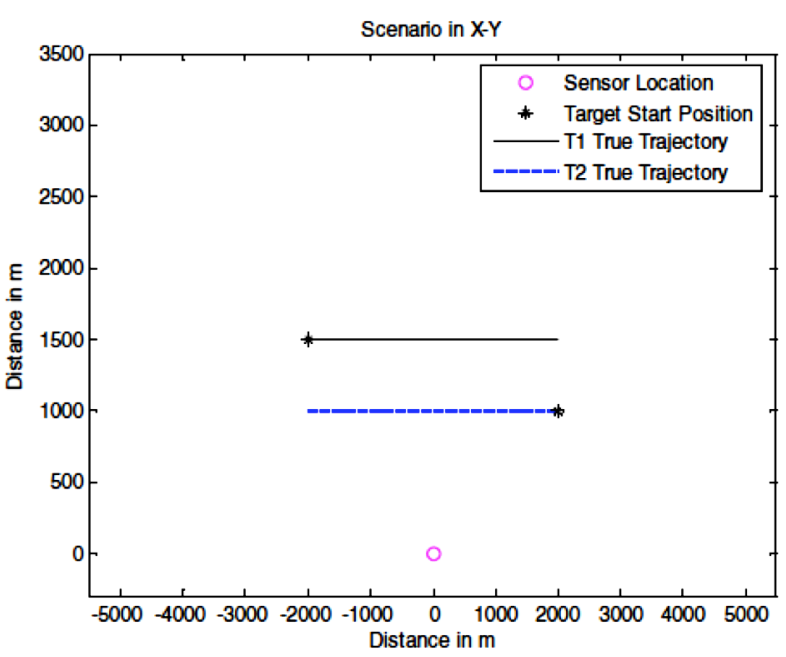

(b)

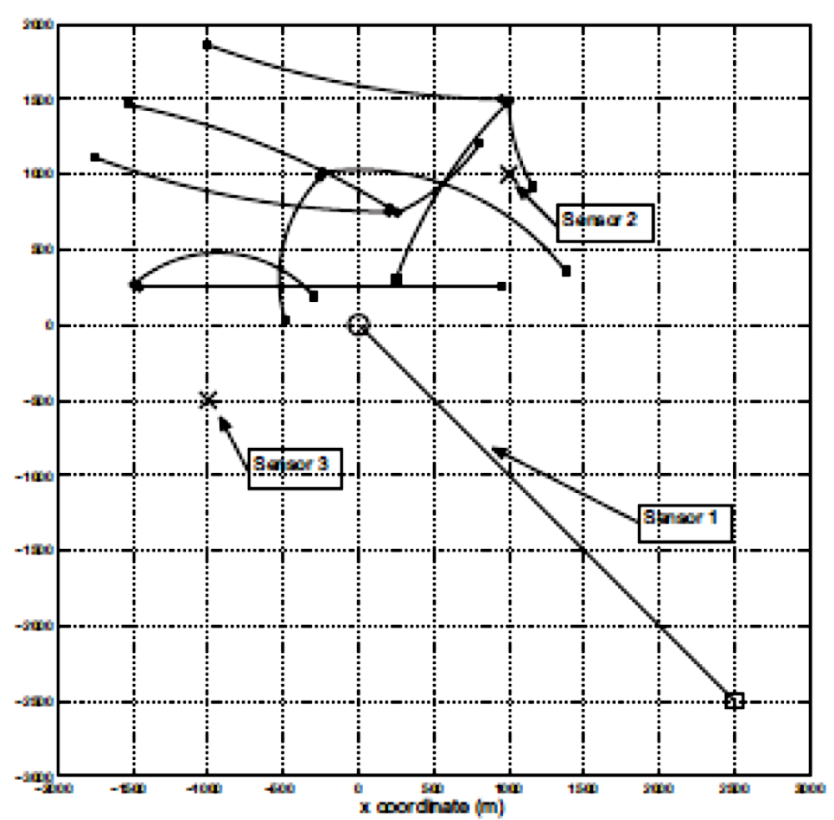

(c) 
Fig.6. Scenarios for multiple targets with single sensor, single target with multiple sensors and multi-target with multiple sensors as shown in [a], [b] and [c] courtesy [105], [76] and [13].

In case of single target with multiple sensors, measurements to track association (M2TA) techniques are used to associate the new measurement to the existing track. For the scenarios involving multiple sensors with single target, track to track association (T2TA) techniques are used to obtain the information pertaining to the particular target. Whereas, for multi-target multi-sensor scenario both the M2TA and T2TA are used. Finally, fusion techniques are used to get the optimized information about the particular target.

\subsection{Batch processing techniques}

Taghavi et al. [88] has discussed about multisensormultitarget for BOT in the presence of position bias. Unlike single sensor BOT, multisensor BOT does not have an observability issue due to the presence of multiple sensors in the surveillance region. The authors have proposed a method to model the bearing only offset bias and the maximum likelihood estimators are used to estimate the biases. In this paper, the scenario is considered with four sensors and sixteen targets. Genetic algorithm (GA), which is a batch of ML estimator was used by authors for optimization problem. The demonstration of the proposed method was performed in the presence of false alarm and missed detection. The sensors send all the measurements to the fusion node and GA is used to obtain the accuracy of bias estimation. The simulation results indicate that, proposed technique is effective, even for a higher noise levels.

\subsection{Recursive nonlinear techniques}

This subsection explains about the different recursive techniques used for single and multisensor- multitarget tracking. V.P.Panakkal et al. [76] has discussed about the technique of derived heading for 2D BOT. The authors stated that, accuracy of state estimate can be increased by deriving the target heading from set of bearing measurements. Two scenarios are considered in this paper to assess the derived heading. Scenario 1 considers single maneuvering target with single sensor and scenario 2 has two targets crossing with respect to bearing measurements with single sensor. In both the scenarios the nonlinear filters EKF and PF are used to estimate the state of the target. For scenario 2 data association technique was used to obtain the information pertaining to the individual targets. The data association in EKF was performed using nearest neighbor data association technique and in PF joint state vector data association technique was used. The simulations were done for both scenarios with $\mathrm{PF}$ and EKF with and without heading and it is compared with CRLB. The results indicate PF shows better performance than EKF in all the cases considered and use of derived heading increases the position and velocity estimates. T. Hanselmann et. al [32] has described an algorithm for tracking of multiple targets using multiple sensors when bearing measurements are asynchronous. Authors have assumed that, the positions of sensor platform are known and it is considered to be passive and observes targets and clutters. Range parameterized unscented Kalman filter (RP-UKF) is used for target detection on the basis of hypothesis, which is known as multiple hypothesis trackers (MHT). In this method, target state is conditioned on each hypothesis to approximate the posterior probability. The track state was divided into two types as best hypothesis and clustered hypothesis and comparison was made between them. The simulation results indicate that state estimate from best hypothesis is good. However during high noise condition the state estimate from clustered hypothesis performs better. Ristic et al. [78], has derived and analyzed theoretical lower bound of the performance of error and proposed three tracking algorithms namely IMM-EKF, IMM-UKF and Multi-mode particle filter (MMPF). The authors have modeled target dynamics using multiple switching dynamic models. These three tracking algorithms were compared with theoretical lower bound. The authors stated that, among three tracking algorithms used, MMPF performs better followed by IMMUKF and finally IMM-EKF. The computational complexity for MMPF is higher than the other two. Based on all these analysis, the authors recommended IMM-UKF for fairly accurate estimates with average computational complexity. F. Hoffman et al. [35] have proposed a trajectory optimization method for two sensor BOT scenario. The authors have assumed that measurements do not contain the identifying feature of the targets. Hence there is a chance of false association with the ghost targets and formation of false trajectories. To reduce this problem authors have multihypothesis tracker (MHT) to find correct hypothesis. The centralized fusion technique is performed and the simulation results reveals that, proposed technique highly resolve the problem of ghost targets and reduces the estimation error. T. Sathyan et.al [81] has discussed the problem of measurement origin uncertainty in case of multiple targets. The authors have used the Multiframe assignment (MFA) algorithm for data association problem. It works by initializing the track for all the measurements in each scan. Then data association was performed between the measurements from current scan and the tracks from previous scan through sliding window format. The UKF in MPC and LPC were used for target detection. From the simulation results the authors have concluded that LPC based filtering performs better. Z. Yan et al [95] has derived and discussed about the 3D triangular ranging formula for two airborne ownships using bearing only measurements from single target. Since BOT is a nonlinear problem, Kalman filter cannot be used directly. The authors stated that using the nonlinear filters like EKF and pseudolinear filter for the nonlinear problem leads to biased estimates. Hence in this paper, the measurements are made linear using 3D triangular passive ranging formula and Kalman filter was implemented. Further, using single model tracking was not enough to handle the target maneuver hence IMM algorithm was used to improve the tracking accuracy. The simulations were performed for both nonmaneuvering and maneuvering target. The result indicates the proposed technique shows better performance with much lower computational complexity. Similarly, Musicki [63] has explained about single maneuvering target trajectory estimation using multiple sensors in bearing only when there exist uncertainty in target positions. Usual method of locating target with multiple sensors involves the method of triangulation. The authors have considered the targets with electronically steered array (ESA) radar which reflects with random direction at random times. In case of passive tracking the tracks are updated at random intervals of time. The target state and measurements are represented as a Gaussian mixture. It is considered that, each track state is a set of track components assuming one measurement component for updating. Hence the process can be done using linear estimators like Kalman filter. When the target takes a maneuver, IMM method is used. The simulation results indicate that, this method is computationally efficient 
and has the disadvantage of exponentially growing number of tracks. Later Musicki [64], has further extended [63] to multiple target scenario. The author has proposed multitarget multi-scan algorithm to identify the unknown existence, number and position of targets in the surveillance region. It is considered that, origin of measurements can be from the tracked target, clutter or from the new target. This paper considers tracking of two targets using three sensors in two dimensional surveillance case. Assuming no prior information for targets position and existence. The probability of detection for each target and sensor was assumed to be 0.5 . The new track is initialized for every measurement. The initial probability for target existence was assumed to be 0.1 . The track is confirmed when probability of track existence reaches the threshold of 0.999 and track is terminated if the probability reaches 0.01 . The simulations were done for both single and multiple targets tracking of the algorithm. The author stated that irrespective of the number of targets tracked computational resource and hence using multi-target of the algorithm is advantageous. S.J. Wong et.al [13] has discussed about the multi-sensor multitarget tracking in BOT. The authors have presented the Gaussian mixture probability density hypothesis (GM-PHD) using EKF and UKF filter. Basically PHD is the first-order statistical moment of the random finite set (RFS), which consists of the finite sets of collections of targets and observations. Simulations were carried out by considering three sensors. The bearing measurement was processed for all the three sensors. The performance of the GM-PHD using EKF and UKF were evaluated using optimal sub-pattern assignment (OSPA) metric. This metric is used to calculate the multi-target miss distance, which indicates the estimation error from the ground truth. From the simulation results, the authors indicate that the proposed technique works better for BOT problems by eliminating the ghost targets. The Table 3 gives the brief overview of the techniques used and its performance analysis used by different authors for category 3.

Table. 3 shows the techniques used and its performance analysis for multi-sensor multi target tracking.

\begin{tabular}{|c|c|c|}
\hline $\begin{array}{c}\text { Authors, reference } \\
\text { and Year }\end{array}$ & Innovation & Performance analysis \\
\hline $\begin{array}{l}\text { T. Hanselmann et al } \\
\text { [32], } 2007\end{array}$ & $\begin{array}{l}\text { 1. Measurement origin hypothesis }(\mathrm{MOH}), 2 . \quad \text { Best } \\
\text { hypothesis state estimate, } \\
\text { 3. Clustering state estimate for multiple target tracking. }\end{array}$ & $\begin{array}{l}\text { RPUKF tracking algorithm was used and better } \\
\text { performance was achieved by clustered state estimate than } \\
\text { best hypothesis state estimate }\end{array}$ \\
\hline $\begin{array}{l}\text { D. Musicki } \\
{[63], 2008}\end{array}$ & $\begin{array}{l}\text { M3T20 and M5T10 model measurements by three and five } \\
\text { component Gaussian mixtures for maneuvering target. }\end{array}$ & $\begin{array}{l}\text { IMM filter was used and it produces better estimation with } \\
\text { lower computational time. }\end{array}$ \\
\hline $\begin{array}{l}\text { D. Musicki } \\
{[64], 2008}\end{array}$ & $\begin{array}{l}\text { 1. Non-Gaussian measurement probability density function } \\
\text { (pdf) by a Gaussian mixture } \\
\text { 2. integrated track splitting (ITS) } \\
\text { 3.integrated probabilistic data association (IPDA) }\end{array}$ & $\begin{array}{l}\text { Algorithms are used for both single and multiple target } \\
\text { cases and better performance was shown for multi-target } \\
\text { scenarios. }\end{array}$ \\
\hline $\begin{array}{l}\text { T. Sathyan et al } \\
\text { [81], } 2010\end{array}$ & $\begin{array}{l}\text { 1.Multiframe assignment algorithm (MFA), 2.lagrangian } \\
\text { relaxation- based suboptimal algorithm }\end{array}$ & MFA using UKF filter in LPC system performs better. \\
\hline $\begin{array}{l}\text { V.P.Panakkal et al } \\
\text { [76], } 2010\end{array}$ & Bearing heading tracking $(\mathrm{BHT})$ & $\begin{array}{l}\text { The use of derived heading by PF shows better } \\
\text { performance compared to EKF. }\end{array}$ \\
\hline $\begin{array}{l}\text { Z. Yan et al } \\
\text { [95], } 2012\end{array}$ & Triangular ranging formula for two airborne platforms & $\begin{array}{l}\text { Triangular ranging formula and IMM-EKF implemented } \\
\text { for both non-maneuvering and maneuvering target } \\
\text { performs better. }\end{array}$ \\
\hline $\begin{array}{l}\text { E. Taghavi et al } \\
\text { [88], } 2016\end{array}$ & $\begin{array}{l}\text { 1. Associated measurement reports (AMR) 2. Genetic } \\
\text { algorithm } \\
\text { 3. proposed bias model }\end{array}$ & $\begin{array}{l}\text { The proposed bias model is effective in handling false } \\
\text { alarm and missed detection and has better performance in } \\
\text { case of high bias values and it is solved using batch ML } \\
\text { estimator. }\end{array}$ \\
\hline $\begin{array}{l}\text { F. Hoffmann et al } \\
\quad[35], 2016\end{array}$ & Trajectory optimization method for multitarget-multisensor. & $\begin{array}{l}\text { Trajectory optimization method using MHT was much } \\
\text { faster and effective in minimizing the ambiguity as well as } \\
\text { track estimation error. }\end{array}$ \\
\hline $\begin{array}{l}\text { S.J. Wong et.al [13], } \\
2011 \\
\end{array}$ & $\begin{array}{l}\text { Square Root Gaussian Mixture PHD filter for Multi-target } \\
\text { BOT }\end{array}$ & $\begin{array}{l}\text { GM-PHD using UKF performs better by eliminating ghost } \\
\text { targets }\end{array}$ \\
\hline
\end{tabular}

\section{Conclusion}

This paper gives the brief review of algorithms used for BOT for three different tracking categories. BOT is the wide area of research used in many radar, sonar, underwater and space surveillance applications. Initially, algorithms used for single sensor single target tracking with constant velocity scenario used in literature are discussed briefly. The algorithms for this scenario were divided into batch and recursive type. The problem of solving the issues related to observability of target state using different algorithms was illustrated. This is followed by brief survey of algorithms used for single sensor and target with constant acceleration. The proposed algorithms for target maneuver detection and tracking, given in the literature were analyzed briefly. Finally, techniques for multiple sensors with single and multiple targets tracking scenario are presented. In case of multi target tracking scenarios, several new algorithms based on hypothesis used are reviewed briefly. The literatures for this scenario are relatively low compared to other two scenarios. In this paper all the algorithms relating to BOT, used in literature are reviewed and improvements to algorithms proposed are reviewed clearly. In future, the MGEKF used for underwater applications has to be implemented for other applications also and the publications related to multi target tracking in BOT has to be increased by implementing new algorithms to get better results with reduced computational time.

This is an Open Access article distributed under the terms of the Creative Commons Attribution License

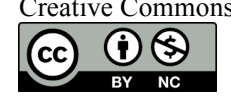




\section{References}

[1] B. D. O. Anderson, J. B. Moore, Optimal Filtering, Prentice Hall, (1979).

[2] V.J. Aidala, S.E. Hammel, "Utilization of Modified Polar Coordinates for Bearings-Only Tracking", IEEE TRANSACTIONS ON AUTOMATIC CONTROL, VOL. AC28, NO. 3, pp. 284-294, MARCH (1983).

[3] S. Arulampalam, B. Ristic, "Comparison of the Particle Filter with Range-Parameterized and Modified Polar EKFs for Angle-Only Tracking", In Signal and Data Processing of Small Targets, Oliver E. Drummond, Editor, Proceedings of SPIE Vol. 4048 , (2000).

[4] S. Arulampalam, B. Ristic, N. Gordon, T. Mansell, "Bearings-Only Tracking of Maneuvering Targets Using Particle Filters", EURASIP Journal on Applied Signal Processing, vol. 15, pp. 2351-2365, (2004).

[5] S. Arulampalam, M. Clark and R. Vinter, "Performance of the Shifted Rayleigh Filter in Single-sensor Bearings-only Tracking",10th International Conference on Information Fusion, 9-12 July (2007).

[6] N. Ashok Kumar and S. Koteswara Rao, "Performance analysis of Modified gain Extended Kalman filter in underwater passive target tracking for stationary and maneuvering observer", International Conference on Electrical, Electronics, Signals, Communication and Optimization (EESCO), 24-25 Jan. (2015).

[7] R. R. Allen, S. S. Blackman, "Angle-only Tracking With a MSC Filter", Digital Avionics Systems Conference, Proceedings, IEEE, pp. 561-566, Los Angeles, CA, October, (1991).

[8] Y. Bar-Shalom, X. Rong Li, T. Kirubarajan, Estimation with Applications to Tracking and Navigation: Theory Algorithms and Software, John Wiley and Sons, Inc. New York, NY, USA, (2001).

[9] Y. Bar-Shalom, P. Willett, and X. Tian, Tracking and Data Fusion: A Handbook of Algorithms, YBS Publishing, (2011).

[10]L. Badriasl, K. Dogancay, "Three-Dimensional Target Motion Analysis Using Azimuth/Elevation Angles," IEEE Transactions On Aerospace And Electronic Systems, vol. 50, no. 4, pp. 31783194, March (2014).

[11] S. Blackman, R. Popoli, Design and Analysis of Modern Tracking Systems, Artech House, (1999).

[12]T. Brehard, J.P. Le Cadre, "Closed-form Posterior Cram'er-Rao Bound for a Manoeuvring Target in the Bearings-Only Tracking Context Using Best-Fitting Gaussian Distribution," IEEE Trans. on Aerospace and Electronic Systems, vol. 42, no. 4, pp. 1198 1223, (2006).

[13]S. Jeffrey Wong, B. Tuong Vo, "Square Root Gaussian Mixture PHD filter for Multi-target bearings only tracking", Seventh International Conference on Intelligent Sensors, Sensor Networks and Information Processing (ISSNIP), pg. 520-525, (2011).

[14]T. Brehard, J.P. Le Cadre, "Hierarchical Particle Filter for Bearings-Only Tracking", IEEE TRANSACTIONS ON AEROSPACE AND ELECTRONIC SYSTEMS, VOL. 43, NO. 4, pp. 1567--1585, OCTOBER (2007).

[15]A. Can Ozelci and R. Vinter,"The Shifted Rayleigh Filter for 3D Bearings-only Measurements with Clutter",15th International Conference on Information Fusion (FUSION) , pp. 1385--1391, 9-12 July (2012)

[16] J.M.C.Clark, S.A. Robbiati and R.B. Vinter, "The Shifted Rayleigh Mixture Filter for Bearings-Only Tracking of Maneuvering Targets",IEEE TRANSACTIONS ON SIGNAL PROCESSING, VOL. 55, NO. 7, pp. 3218--3226, JULY (2007).

[17]C. Carvalho, M. Johannes, H. Lopes et al., "Particle Learning and Smoothing",Statistical Science, vol. 25, no. 1, pp. 88--106, (2010).

[18]K. Dogancay, "On the efficiency of a bearings-only instrumental variable estimator for target motion analysis", Signal Processing, Elsevier, Vol. 85, no. 3, pp. 481--490, (2005).

[19]A. Farina, "Target tracking with bearings-Only measurements", Signal Processing, Elsevier, vol. 78, no. 1, pp. 61-78, (1999).

[20]D.Franken, "Filter Initialization and Batch Estimation for Tracking with Angular-Only Measurements",18th International Conference on Information Fusion, pp. 1542--1549, Washington, DC, July 69, (2015).

[21]E. Fogel and M. Gavish, "Nth order dynamics Target Observability From Angle Measurements," IEEE TRANSACTIONS ON
AEROSPACE AND ELECTRONIC SYSTEMS, VOL. 24, NO. 3, pp. 305--308, (1988).

[22]P.J.Galkowski and M.A.Islam, "An Alternative Derivation of the Modified Gain Function of Song and Speyer," IEEE TRANSACTIONS ON AUTOMATIC CONTROL, VOL. 36, NO. 11, pp. 1323--1326, (1991).

[23] A.Gelb, Editor, Applied Optimal Estimation, MIT Press, (1974).

[24]W.Grossman, "Bearings-Only Tracking: A Hybrid Coordinate System Approach", IEEE conference on Decision and Control, pp. 2032 - 2037, Brighton, Dec 11-13, (1991).

[25]N.Gordon and M.Pitt, "A comparison of sample based filters and the Extended Kalman filter for the bearings-only target problem",9th European Signal Processing Conference, EUSIPCO, 8-11 Sept. (1998).

[26]N.Gordon, D.Salmond and A.Smith, "Novel approach to nonlinear/non-Gaussian Bayesian state estimation", Proceedings of the IEE, VOL.140, NO.2, pp.107-113, Apr. (1993).

[27]Y.Guo, A.Xue and D.Peng, "A recursive algorithm for bearingsonly tracking with signal time delay",Signal Processing Elsevier, Vol.88, No.6, pp. 1539-1552, (2008).

[28]R.Gupta, A. Kumar, I. N. Kar and R. Bahl, "Bearings-only tracking of non-maneuvering target with missing bearings data", Underwater Technology (UT),IEEE, 23-25 Feb, (2015).

[29] S.D. Gupta, J.Y. Yu, M. Mallick, M. Coates, and M. Morelande, "Comparison of Angle-only Filtering Algorithms in 3D Using EKF, UKF, PF, PFF, and Ensemble KF,"Proc. 18th International Conference on Information Fusion, pp. 1649--1656, Washington DC, USA, July 6-9, (2015).

[30] S. E. Hammel, V. J. Aidala, "Observability requirements for three dimensional tracking via angle measurements", IEEE Trans. Aerosp. Electron. Syst, vol.21, no. 2, pp. 200-207, (1985).

[31]S. E. Hammel, P.T. Liu, E.J. Hilliard, et.al, "Optimal observer motion for localization with bearing measurements", Computers and Mathematics with applications, vol. 18, no. 1-3, pp. 171-180, (1989).

[32] T. Hanselmann and M. Morelande, "Multiple Target Tracking With Asynchronous Bearings-Only-Measurements",10th International Conference on Information Fusion, 9-12 July (2007).

[33]J.P. Helferty, D.R. Mudgett and J.E.Dzielski, "Trajectory Optimization for Minimum Range Error in Bearings-only Source Localization", Proceedings of Engineering in Harmony with Ocean (OCEANS), 18-21 Oct. (1993).

[34] M.L. Hernandez, B.Ristic and A.Farina, "A Performance Bound for Maneuvering Target Tracking Using Best-Fitting Gaussian Distributions",8th International Conference on Information Fusion, 25-28 July (2005).

[35]F. Hoffmann, A. Charlish and W. Koch, "Trajectory Optimization for Multi-Platform Bearing-Only Tracking with Ghosts",19th International Conference on Information Fusion, Heidelberg, Germany - July 5-8, (2016).

[36]J.Horst and M. Oispuu, "Target Localization and Course Change Detection Using Bearing and Bearing Rate Measurements", Proceedings of the 14th International Conference on Information Fusion (FUSION), 5-8 July, pp. 1-8, (2011).

[37] G.P. Huang, K.X. Zhou, N. Trawny, et.al, "Bearing-only Target Tracking using a Bank of MAP Estimators",IEEE International Conference on Robotics and Automation, pp. 4998--5005, Shanghai, China, May 9-13, (2011).

[38] Y.Huang and T.L. Song, "Iterated Modified Gain Extended Kalman Filter with Applications to Bearings Only Tracking", Journal of Automation and Control Engineering, Vol. 3, No. 6, pp. 475-479, December (2015).

[39]A.H. Jazwinski, Stochastic processes and filtering theory, Courier Corporation, (2007).

[40] S.J.Julier and J.K.Uhlmann, "Unscented Filtering and Nonlinear Estimation", PROCEEDINGS OF THE IEEE, VOL. 92, NO. 3, MARCH (2004).

[41]R. Karlsson and F. Gustafsson, " Range estimation using angle-only target tracking with particle filters," Proc. American Control Conference, pp. 3743 -- 3748, (2001).

[42] T.R. Kronhamn, 'Bearings-only target motion analysis based on a multihypothesis Kalman filter and adaptive ownship motion control," IEE Proc.-Radar, Sonar Navigation, Vol. 145, No. 4, August 1998. 
[43]T.R.Kronhamn, "Angle-Only Tracking of Manoeuvring Targets using Adaptive-IMM Multiple Range Models",Proc. of the IEE International Radar Conference, Edinburgh, UK, pp. 310--314, oct. (2002).

[44] T.Kronhamn, "Angle-Only Tracking of Manoeuvring Targets using Double Sets of Multiple Range Models and Probability Mass Diffusion",Information, Decision and Control, pp. 41--46, 12-14 Feb. (2007).

[45]T. Kirubarajan, Y. Bar-shalom and D. Lerro, "Bearings-Only Tracking of Maneuvering Targets Using a Batch-Recursive Estimator," IEEE TRANSACTIONS ON AEROSPACE AND ELECTRONIC SYSTEMS, VOL. 37, NO. 3, pp. 770--780, JULY (2001).

[46] S.Koteswara Rao, "Algorithm for detection of manoeuvring targets in bearings-only passive target tracking", IEE Proc.Radar Sonar Navigation, Vol. 146, No.3, pp. 141--146, June (1999).

[47] S. Koteswara Rao, "Modified gain extended Kalman filter with application to bearings-only passive maneuvering target tracking",IEE Proc.-Radar Sonar Navigation, Vol. 152, No. 4, pp. 239--244, August (2005).

[48] S. Koteswara Rao and V. Sunanda Babu, "Unscented Kalman Filter With Application To Bearings-Only Passive Manoeuvring Target Tracking," IEEE-International Conference on Signal processing, Communications and Networking, Anna University Chennai India, Jan 4-6, pp. 219-224, (2008).

[49]Q. Li, F. Guo et al., "Research of Satellite-to-Satellite Passive Tracking Using Bearings-Only Measurements In J2000 ECI Frame", International Conference on Radar, 16-19 Oct. (2006).

[50]Q. Li, F. Guo, Y. Zhou, and W. Jiang, “'Observability of Satellite to Satellite Passive Tracking from Angles Measurements", Proc. IEEE International Conference on Control and Automation, pp. 1926 -- 1931, (2007).

[51]H. Li, L. Chen and A. Sheng, "Two-Step Modeling and Observability for Bearings-Only Tracking," International Conference on Mechatronics and Automation, IEEE, , August 9 12, Changchun, China, pp. $691-695,(2009)$.

[52] J.Liu, Y.Wang and Z.Wang, "A Novel Hybrid Estimator for RealTime Bearings-Only Target Tracking," Control and Decision Conference (CCDC), China, pp. 3900-3905, 28-30 May (2016).

[53]J.Liu, Z.Wu, J.Yang and Z.Li, "A New Algorithm for BearingsOnly Parametric Trajectory Tracking," Advanced in Control Engineering and Information Science, Procedia Engineering, Vol.15, pp.2644 -- 2649, (2011).

[54]J.P.Le Cadre, S. Laurent-Michel, "Optimizing the receiver maneuvers for bearings-only tracking",Automatica, Vol. 35, No. 4, pp. 591-606, (1999).

[55]J.P.Le Cadre, O.Tremois, "Bearings-Only Tracking for Maneuvering Sources", IEEE TRANSACTIONS ON AEROSPACE AND ELECTRONIC SYSTEMS, VOL. 34, NO. 1, pp. 179--193, (1998).

[56]J.P.Le Cadre, C. Jauffret, "Discrete-Time Observability and Estimability Analysis for Bearings-Only Target Motion Analysis", IEEE TRANSACTIONS ON AEROSPACE AND ELECTRONIC SYSTEMS, Vol. 33, No. 1, pp. 178--201, January (1997).

[57]P.H.Leong, S.Arulampalam, T.A.Lamahewa, T.D.Abhayapala, "A Gaussian-Sum Based Cubature Kalman Filter for Bearings-Only Tracking", IEEE TRANSACTIONS ON AEROSPACE AND ELECTRONIC SYSTEMS, Vol.49, No.2, pp. 1161--1176, (2013).

[58]A. Logothetis, A. Isaksson and R.J. Evans, "Comparison of Suboptimal Strategies for Optimal Own-Ship Maneuvers in Bearings-Only Tracking", Proceedings of the American Control Conference, Philadelphia, Pennsylvania, June (1998).

[59]M. Mallick and S.Arulampalam, "Comparison of Nonlinear Filtering Algorithms in Ground Moving Target Indicator (GMTI) Target Tracking", Proceedings of SPIE, San Diego, CA, Aug. (2003).

[60] M. Mallick, L. Mihaylova, S. Arulampalam, and Y. Yan, “Angleonly Filtering in 3D Using Modified Spherical and Log Spherical Coordinates", Proc. 14th International Conference on Information Fusion, Chicago, USA, July 5-8, (2011).

[61] M. Mallick, M. Morelande , L. Mihaylova, S. Arulampalam, and Y. Yan, "Comparison of angle-only filtering algorithms in 3D using Cartesian and modified spherical coordinates,"Proc. 15th International Conference on Information Fusion, pp. 1392--1399, Singapore, July 9--12, (2012).

[62] M. Mallick, M. Morelande, L. Mihaylova, S. Arulampalam, and Y. Yan, Angle-only filtering in three dimensions, Ch. 1, in
Integrated Tracking, Classification, and Sensor Management: Theory and Applications, Eds. M. Mallick, V. Krishnamurthy, and B.-N. Vo, Ed., Wiley/IEEE, pp. 3-42, December (2012).

[63]D. Musicki, "Bearings only multi-sensor maneuvering target tracking", Systems and Control Letters, Vol. 57, pp. 216 - 221, (2008).

[64]D. Musicki, "Multi-Target Tracking using Multiple Passive Bearings-Only Asynchronous Sensors", IEEE TRANSACTIONS ON AEROSPACE AND ELECTRONIC SYSTEMS, Vol.44, No.3, pp. 1151--1160, (2008).

[65]A.K. Mohammadiyan and H.Khaloozadeh, "Adaptive IMMPF for Bearing-Only Maneuvering Target Tracking in Wireless Sensor Networks", 4th International Conference on Control, Instrumentation, and Automation (ICCIA), Qazvin, Iran. pp. 6-11, 27-28 January (2016).

[66] M.R. Morelande, "A Marginalised particle filter for bearings only tracking",IEEE International Conference on Acoustics, Speech and Signal Processing (ICASSP), 26-31 May 2013, pp. 6298-6302, (2013).

[67]L.Meiqin, Z.Di and Z.Senlin, "Bearing-Only Target Tracking using Cubature Rauch-Tung-Striebel Smoother", Proceedings of the 34th Chinese Control Conference, Hangzhou, China, pp.4734-4738, July 28-30, (2015).

[68] S. C. Nardone, V. J. Aidala, "Observability criteria for bearingsonly target motion analysis," IEEE Trans. Aerosp. Electron. Syst, vol.17, no. 2, pp. 162-166, Oct. (1981).

[69] S.C.Nardone, A.G.Lindgren and K.F.Gong, "Fundamental Properties and Performance of Conventional Bearings-Only Target Motion Analysis", IEEE TRANSACTIONS ON AUTOMATIC CONTROL, vol.29, no.9, pp. 775--787, September (1984).

[70] S.C.Nardone, "A Closed-Form Solution to Bearings-Only Target Motion Analysis", IEEE JOURNAL OF OCEANIC ENGINEERING, VOL. 22, NO. 1, pp. 168--178, JANUARY (1997).

[71]C. Nemeth, P. Fearnhead, L. Mihaylova, et.al, "Bearings-Only Tracking with Particle Filtering for Joint Parameter Learning and State Estimation," 15th International Conference on Information Fusion (FUSION), pp. 824--831, 9-12 July (2012).

[72]L. Qiang, S. Lihui, W. Hongxian, G. Fucheng, "Utilization of Modified Spherical Coordinates for Satellite to Satellite Bearings-Only Tracking," Chinese Journal of space science, pp. 627-634, (2009).

[73]Z.Qian and T.L.Song, "Improvement of Bearings Only Target Tracking Using Smoothing", IEEE International Conference on Control Science and Systems Engineering (CCSSE), pp. 1--5, 29-30 Dec. (2014).

[74]N. Peach, "Bearings-only tracking using a set of rangeparameterised extended Kalman filters," IEE Pror.-Control Theory Applications, Vol. 142, No.1, pp. 73--80, January (1995).

[75]A.N. Payne, "Observability conditions for angles-only tracking", Twenty-Second Asilomar Conference on Signals, Systems and Computers, pp. 451--457, 31 Oct.-2 Nov. (1988).

[76] V.P.Panakkal, R.Velmurugan, "Bearings-Only Tracking using Derived Heading", IEEE Aerospace Conference, 6-13 March, USA, (2010).

[77]B. Ristic, S. Arulampalam, N. Gordon, Beyond the Kalman Filter: Particle Filters for Tracking Applications, Artech House, (2004).

[78]B. Ristic, S. Arulampalam, "Tracking a maneuvring target using angle-only measurements:algorithms and performance", Signal Processing, Vol. 83, pp. 1223--1238, (2003).

[79]B. Ristic, S. Arulampalam, "Bernoulli Particle Filter with Observer Control for Bearings-Only Tracking in Clutter", IEEE TRANSACTIONS ON AEROSPACE AND ELECTRONIC SYSTEMS, VOL. 48, NO. 3, pp. 2405--2415, (2012).

[80]X. Rong Li, Z. Zhao and X. Li, "General Model-Set Design Methods for Multiple-Model Approach", IEEE TRANSACTIONS ON AUTOMATIC CONTROL, VOL. 50, NO. 9, pp. 1260--1276, (2005).

[81]T. Sathyan, A. Sinha and M. Mallick, "A Multiframe Assignment Algorithm for Single Sensor Bearings-Only Tracking",13th Conference on Information Fusion (FUSION) , 26-29 July (2010).

[82] S. Sadhu, S. Mondal et.al., "Sigma point Kalman filter for bearing only tracking", Signal processing, Elsevier, Vol.86, No.12, pp. 3769-3777, December (2006).

[83]B.L. Scala and M. Morelande, "An analysis of the single sensor bearings-only tracking problem," 11 th International Conference on Information Fusion, pp. 1-6. IEEE, (2008). 
[84]T.L. Song and J.L. Speyer, "A stochastic Analysis of a modified gain extended kalman filter with applications to estimation with bearing only measurements," IEEE Trans. Automatic Control, Vol.30, No.10, pp. 940--949, (1985).

[85] T.L. SONG, "Observability of Target Tracking with Bearings-Only Measurements," IEEE TRANSACTIONS ON AEROSPACE AND ELECTRONIC SYSTEMS, VOL. 32, NO.4, (1996).

[86]D.V. Stallard, "Angle-only tracking filter in modified spherical coordinates," Journal of Guidance, Control, and Dynamics, vol. 14, no. 3, pp. 694--696, (1991).

[87]O. Straka, J. Dunik and M. Simandl, "Performance Evaluation of Local State Estimation Methods in Bearings-only Tracking Problems," 14th International Conference on Information Fusion, Chicago, Illinois, USA, July 5-8, (2011).

[88]E. Taghavi, R. Tharmarasa and T. Kirubarajan, "MultisensorMultitarget Bearing-Only Sensor Registration", IEEE Transactions on Aerospace and Electronics Systems, vol. 52, no. 4, pp. 1654 - 1666, March (2016).

[89]P.Tichavsky, C.H.Muravchik and A.Nehorai, "Posterior CramerRao Bounds for Discrete-Time Nonlinear Filtering", IEEE TRANSACTIONS ON SIGNAL PROCESSING, VOL. 46, NO. 5, pp. 1386--1396, MAY (1998).

[90]X. Wang, M. Morelande and B. Moran, "Target Motion Analysis Using Single Sensor Bearings-Only Measurements", 2nd International Congress on Image and Signal Processing, 30 October (2009).

[91] M. West and J. Liu, "“Combined parameter and state estimation in simulation-based filtering", Sequential Monte Carlo Methods in Practice, A. Doucet, N. de Freitas, and N. Gordon, Eds. New York: Springer-Verlag, pp. 197-223, (2001).

[92]H.Wu, S.Chen, et.al, "Range-parameterized orthogonal simplex cubature Kalman filter for bearings-only measurements", IET Science, Measurement and Technology, Vol.10, No.4, pp. 370 374, (2016).

[93]B.Xu, Z.Wu and Z.Wang, "On the Crame'r-Rao lower bound for biased bearings-only maneuvering target tracking", Signal Processing, Elsevier, Vol.87, No.12, pp.3175-3189, (2007).

[94]H. Pesonen, R. Piche, "Cubature-based Kalman Filters for Positioning", $7^{\text {th }}$ workshop on Positioning, Navigation and Communication, pp. 1-5, (2010).

[95]Z. Yan and L. Jianxun, "Interactive Multiple Kalman Filters based 3-D Target Tracking for Two Airborne Platforms with BearingsOnly Measurements",24th Chinese Control and Decision Conference (CCDC), 23-25 May (2012).
[96]Z. Zhao, X. Rong Li, "The Behavior of Model Probability in Multiple Model Algorithms",7th International Conference on Information Fusion (FUSION), vol. 1, pp.331-336, (2005).

[97] Y.J. Zhang and G.Z. Xu, "Bearings-Only Target Motion Analysis via Instrumental Variable Estimation", IEEE TRANSACTIONS ON SIGNAL PROCESSING, VOL. 58, NO. 11, pp. 5523--5533, NOVEMBER (2013).

[98]Q.Zhang, S.H.Park and T.L.Song, "Improved 3D Angle-only Target Tracking with Smoothing", International Conference on Control, Automation and Information Sciences (ICCAIS), Ansan, Korea, pp. 238--243, (2016).

[99] W. Zhu, Z. Xu, et.al, "Research on the Observability of Bearingsonly Target Tracking Based on Multiple Sonar sensors", Second International Conference on Instrumentation and Measurement, Computer, Communication and Control, IEEE, 8-10 Dec. (2012).

[100] L. Zuo, R.Niu, and P.K. Varshney, "Conditional Posterior Cramér-Rao Lower Bounds for Nonlinear Sequential Bayesian Estimation", IEEE TRANSACTIONS ON SIGNAL PROCESSING, VOL. 59, NO. 1, pp. 1--14, JANUARY (2011).

[101] F. Bavencoff, J.M. Vanpeperstraete and J.P. Le Cadre, "Constrained-Bearings-only target motion analysis via Markov Chain Monte Carlo methods", IEEE Transactions on Aerospace and electronic systems, Vol. 42, No.4, pp. 1240-1263, October (2006).

[102] E. Tom Northardt, I. Bilik and Y. Abramovich, "Bearings-Only Constant velocity target maneuver detection via Expected likelihood", IEEE Transactions on Aerospace and electronic systems, Vol. 50, No. 4, pp. 2974-2988, October (2014).

[103] H. Seung Son, J. Bae Park and Y. Hoon joo, "Fuzzy c-meansbased intelligent tracking algorithm for an underwater maneuvering target”, IET Radar Sonar Navigation, Vol. 8, No. 9, pp. 1042-1050, (2014).

[104] L. Qun Li, W. Xin Xie and Z. Xiang Liu, "Auxiliary Truncated Particle filtering with Least-square method for Bearings-Only maneuvering target tracking", IEEE Transactions on Aerospace and electronic systems, Vol. 52, No. 5, October (2016).

[105] A.G. Lindgren, K.F. Gong, "Position and Velocity Estimation Via Bearing Observations", IEEE Transactions on Aerospace and electronic systems, Vol. 14, No.4, (1978).

[106] H.D. Hoelzer, "Range normalized coordinates for optimal AngleOnly tracking in three dimensions", Technical report, November (1980). 